CIRJE-F-1009

\title{
An Asymptotic Expansion for Forward-Backward SDEs: A Malliavin Calculus Approach
}

\author{
Akihiko Takahashi \\ The University of Tokyo \\ Toshihiro Yamada \\ Hitotsubashi University
}

April 2016

CIRJE Discussion Papers can be downloaded without charge from:

http://www.cirje.e.u-tokyo.ac.jp/research/03research02dp.html

Discussion Papers are a series of manuscripts in their draft form. They are not intended for circulation or distribution except as indicated by the author. For that reason Discussion Papers may not be reproduced or distributed without the written consent of the author. 


\title{
An asymptotic expansion for forward-backward SDEs: a Malliavin calculus approach ${ }^{* \dagger}$
}

\author{
Akihiko Takahashi ${ }^{\ddagger}$ and Toshihiro Yamada ${ }^{\S}$
}

September 10, 2016

\begin{abstract}
This paper proposes a new analytical approximation scheme for the representation of the forwardbackward stochastic differential equations (FBSDEs) of Ma and Zhang (2002). In particular, we obtain an error estimate for the scheme applying Malliavin calculus method for the forward SDEs combined with the Picard iteration scheme for the BSDEs. We also show numerical examples for pricing option with counterparty risk under local and stochastic volatility models, where the credit value adjustment (CVA) is taken into account.
\end{abstract}

Keywords: Forward-Backward Stochastic Differential Equations (FBSDEs), Asymptotic expansion, Malliavin calculus, CVA, Local volatility model, Stochastic volatility model

\section{Introduction}

In this paper, we propose a new asymptotic expansion scheme with its error estimate for the forwardbackward stochastic differential equations (FBSDEs). As an application, we derive a recursive expansion formula for option prices with CVA under local and stochastic volatility models and show numerical examples.

Bismut [1] introduced the backward stochastic differential equations (BSDEs) for the linear case, and Pardoux and Peng [28] initiated the study for the non-linear BSDEs. Since then, in addition to its theoretical researches, substantial numbers of numerical schemes for the solutions to the BSDEs have been proposed. The one of the main reasons is that the BSDEs are closely related to various valuation problems in finance (e.g. pricing securities under asymmetric/imperfect collateralization, optimal portfolio and indifference pricing issues in incomplete and/or constrained markets, modeling credit risks). Their financial applications are discussed in details for example, El Karoui et al. [7], Ma and Yong [22], a recent book edited by Carmona [2] and references therein.

Although a large number of finite difference methods and simulation-based methods were proposed for numerical approximations of the solutions to BSDEs, their analytical approximation methods have been rarely discussed. Fujii and Takahashi [8], [9], [12], Fujii et. al. [13] are exceptions, where they presented a simple analytical approximation with perturbation or/and interacting particle scheme for non-linear fully coupled FBSDEs without error estimate. Especially, Fujii and Takahashi [9] derived an approximation formula for dynamic optimal portfolio in an incomplete market with stochastic volatility, and confirmed its validity through numerical experiment.

This paper presents a new analytical approximation method for the FBSDEs based on a Picard-type iteration and an asymptotic expansion (for the asymptotic expansion approach, see Takahashi and Yamada [33] [34] and related previous works [30][24][31][35][29] for example). Also, our method can be regarded as an extension of the representation theorem of BSDEs by Ma and Zhang [23]. Ma and Zhang's result is known as the gradient representation of BSDEs without differentiation, i.e. for a system of BSDE

$$
X_{s}^{t, x}=x+\int_{t}^{s} b\left(u, X_{u}^{t, x}\right) d u+\sum_{j=1}^{d} \int_{t}^{s} \sigma_{j}\left(u, X_{u}^{t, x}\right) d W_{u}^{j},
$$

${ }^{*}$ Forthcoming in Asia-Pacific Financial Markets.

${ }^{\dagger}$ This research is supported by JSPS KAKENHI (Grant Number 25380389, 16K13773).

${ }^{\ddagger}$ University of Tokyo

${ }^{\S}$ Hitotsubashi University 


$$
Y_{s}^{t, x}=g\left(X_{T}^{t, x}\right)+\int_{s}^{T} f\left(u, X_{u}^{t, x}, Y_{u}^{t, x}, Z_{u}^{t, x}\right) d u-\int_{s}^{T} Z_{u}^{t, x} d W_{s},
$$

where $f, g$ are assumed to be only Lipschitz continuous, [23] showed the formula for the gradient of

$$
u(t, x)=Y_{t}^{t, x}=E\left[g\left(X_{T}^{t, x}\right)+\int_{s}^{T} f\left(u, X_{u}^{t, x}, Y_{u}^{t, x}, Z_{u}^{t, x}\right) d u\right]
$$

as

$$
\partial_{x} u(t, x) \sigma(t, x)=Z_{t}^{t, x}=E\left[g\left(X_{T}^{t, x}\right) N_{T}^{t, x}+\int_{s}^{T} f\left(u, X_{u}^{t, x}, Y_{u}^{t, x}, Z_{u}^{t, x}\right) N_{u}^{t, x} d u\right] \sigma(t, x)
$$

where $N_{u}^{t, x}=\frac{1}{u-t} \int_{t}^{u} \sigma^{-1}\left(X_{v}^{t, x}\right) \partial_{x} X_{v}^{t, x} d W_{v}$ is a Malliavin weight. Then by Ma and Zhang's result, BSDE $\left(Y_{s}^{t, x}, Z_{s}^{t, x}\right)=\left(u\left(s, X_{s}^{t, x}\right), \partial_{x} u\left(s, X_{s}^{t, x}\right) \sigma\left(s, X_{s}^{t, x}\right)\right)$ is represented without derivatives of parameters $f$ and $g$.

We expand this representation of BSDE by a perturbation method to obtain an analytical approximation. Roughly speaking, for a perturbed forward $\operatorname{SDE} X_{s}^{\varepsilon, t, x}, \varepsilon \in(0,1]$ and an associated backward $\operatorname{SDE}\left(Y_{s}^{\varepsilon, t, x}, Z_{s}^{\varepsilon, t, x}\right)$ of the form

$$
\begin{aligned}
X_{s}^{\varepsilon, t, x} & =x+\int_{t}^{s} b\left(u, X_{u}^{\varepsilon, t, x}\right) d u+\varepsilon \sum_{j=1}^{d} \int_{t}^{s} \sigma_{j}\left(u, X_{u}^{\varepsilon, t, x}\right) d W_{u}^{j} \\
Y_{s}^{\varepsilon, t, x} & =g\left(X_{T}^{\varepsilon, t, x}\right)+\int_{s}^{T} f\left(u, X_{u}^{\varepsilon, t, x}, Y_{u}^{\varepsilon, t, x}, Z_{u}^{\varepsilon, t, x}\right) d u-\int_{s}^{T} Z_{u}^{\varepsilon, t, x} d W_{s},
\end{aligned}
$$

we show the following recursive asymptotic expansion around a Gaussian model $\bar{X}_{s}^{t, x}=X_{s}^{0, t, x}+\left.\varepsilon \frac{\partial}{\partial \varepsilon} X_{s}^{\varepsilon, t, x}\right|_{\varepsilon=0}$ : i.e. for $k \geq 0, N \geq 1$

$$
\begin{aligned}
Y_{t}^{\varepsilon, t, x} & \simeq u^{\varepsilon, k+1, N}(t, x)=E\left[g\left(\bar{X}_{T}^{t, x}\right)+\int_{t}^{T} f\left(s, \bar{X}_{s}^{t, x}, Y_{s}^{\varepsilon, k, N, t, x}, Z_{s}^{\varepsilon, k, N, t, x}\right) d s\right] \\
& +\sum_{i=1}^{N} \varepsilon^{i} E\left[g\left(\bar{X}_{T}^{t, x}\right) \pi_{i, T}^{t, x}+\int_{t}^{T} f\left(s, \bar{X}_{s}^{t, x}, Y_{s}^{\varepsilon, k, N, t, x}, Z_{s}^{\varepsilon, k, N, t, x}\right) \pi_{i, s}^{t, x} d s\right], \\
Z_{t}^{\varepsilon, t, x} & \simeq\left(\partial_{x} u^{\varepsilon, k+1, N} \sigma\right)(t, x)=\left\{E\left[g\left(\bar{X}_{T}^{0, t, x}\right) N_{0, T}^{t, x}+\int_{t}^{T} f\left(s, \bar{X}_{s}^{t, x}, Y_{s}^{\varepsilon, k, N, t, x}, Z_{s}^{\varepsilon, k, N, t, x}\right) N_{0, s}^{t, x} d s\right]\right. \\
& \left.+\sum_{i=1}^{N} \varepsilon^{i} E\left[g\left(\bar{X}_{T}^{0, t, x}\right) N_{i, T}^{t, x}+\int_{t}^{T} f\left(s, \bar{X}_{s}^{t, x}, Y_{s}^{\varepsilon, k, N, t, x}, Z_{s}^{\varepsilon, k, N, t, x}\right) N_{i, s}^{t, x} d s\right]\right\} \varepsilon \sigma(t, x),
\end{aligned}
$$

where $Y_{s}^{\varepsilon, k, N, t, x}=u^{\varepsilon, k, N}\left(s, \bar{X}_{s}^{t, x}\right)$ and $Z_{s}^{\varepsilon, k, N, t, x}=\partial_{x} u^{\varepsilon, k, N} \sigma\left(s, \bar{X}_{s}^{t, x}\right)$ with a usual asymptotic expansion $\left(u^{\varepsilon, 0, N}, \partial_{x} u^{\varepsilon, 0, N} \sigma\right)$ and the processes $\pi_{i, s}^{t, s}, i=1, \cdots, N$ and $N_{i, s}^{t, x}, i=0,1, \cdots, N$ are Malliavin weights for the expansion. Applying properties of so called Kusuoka-Stroock functions introduced by Kusuoka [18], we obtain an error estimate of our scheme.

The organization of this paper is as follows: The next section describes an idea for our method using a well-known example. Section 3 generalizes the idea and summarizes our algorithm in a general setting. After Section 4 provides the notations and basic results used in later sections, Section 5 presents our main result with its proof. Applying our scheme, Section 6 provides a simple numerical example for pricing option with counterparty risk under local volatility and stochastic volatility models. Section 7 concludes.

\section{Motivated example}

In this section, we show an idea for our approximation method using the BSDE appearing in a well-known example of mathematical finance, so called "hedging claims with higher interest rate for borrowing" (e.g. [7], Cvitanic and Karatzas [3]).

Specifically, let us consider the following FBSDE:

$$
\begin{aligned}
d X_{t} & =\mu X_{t} d t+\sigma X_{t} d W_{t} \\
X_{0} & =x_{0} \\
d Y_{t} & =r Y_{t} d t-f\left(Y_{t}, Z_{t}\right) d t+Z_{t} d W_{t} \\
Y_{T} & =g\left(X_{T}\right)=\max \left(X_{T}-K_{1}, 0\right)-2 \max \left(X_{T}-K_{2}, 0\right),
\end{aligned}
$$


where $f(y, z)=(R-r) \max \left(\frac{z}{\sigma}-y, 0\right)-\left(\frac{\mu-r}{\sigma}\right) z$. When the borrowing rate $R$ is higher than the lending rate $r$ (i.e. $R>r$ ), the solution to the FBSDE above, $Y=\left\{Y_{t}: 0 \leq t \leq T\right\}$ represents the value process of a self-financing hedging strategy for a target payoff given by $g\left(X_{T}\right)$, and $Z$ stands for the hedging strategy where $Z_{t} / \sigma$ is the amount invested at time $t$ in the risky asset whose price process is given by $S .^{1}$ In particular, we note that the specification of $g\left(X_{T}\right)$ as an option spread creates both lending and borrowing in the strategy. Here, $r, R, \mu$ and $\sigma$ are assumed to be positive constants.

$Y=\left(Y_{t}\right)_{t \in[0, T]}$ is represented as the following non-linear expectation:

$$
Y_{t}=e^{-r(T-t)} E\left[g\left(X_{T}\right) \mid \mathcal{F}_{t}\right]+e^{-r(T-t)} E\left[\int_{t}^{T} f\left(Y_{u}, Z_{u}\right) d u \mid \mathcal{F}_{t}\right],
$$

where $\left(\mathcal{F}_{t}\right)_{t}$ is the filtration generated by $W$, i.e., $\mathcal{F}_{t}=\sigma\left(W_{s} ; s \leq t\right), t \in[0, T]$. We denote by $\left(X^{t, x}, Y^{t, x}, Z^{t, x}\right)$ the adapted solution to the FBSDE's (9) and (10) restricted to $[t, T]$ with $X_{t}^{t, x}=x$, a.s. Next, define $u$ as

$$
u(t, x):=Y_{t}^{t, x}=e^{-r(T-t)} E\left[g\left(X_{T}^{t, x}\right)\right]+e^{-r(T-t)} E\left[\int_{t}^{T} f\left(Y_{u}^{t, x}, Z_{u}^{t, x}\right) d u\right] .
$$

Then, using this $u, Z=\left(Z_{t}\right)_{t \in[0, T]}$ is obtained as follows:

$$
Z_{t}=\sigma X_{t} \frac{\partial}{\partial x} u\left(t, X_{t}\right)
$$

Moreover, applying a representation result by [23], one has

$$
Z_{t}^{t, x}=e^{-r(T-t)}\left\{E\left[g\left(X_{T}^{t, x}\right) N_{T}^{t, x}\right]+E\left[\int_{t}^{T} f\left(Y_{u}^{t, x}, Z_{u}^{t, x}\right) N_{u}^{t, x} d u\right]\right\} \sigma x
$$

where $N^{t, x}=\left(N_{s}^{t, x}\right)_{s \in[t, T]}$ is the Malliavin weight process given $X_{t}=x, t \in[0, T]$ :

$$
N_{u}^{t, x}=\frac{1}{u-t} \int_{t}^{u} \sigma^{-1}\left(X_{\tau}^{t, x}\right) \frac{\partial}{\partial x} X_{\tau}^{t, x} d W_{\tau} .
$$

Next, let us show an example of an analytical approximation for the BSDE using the Picard-type iteration. In the first place, define $u^{0}(t, x)$ as

$$
u^{0}(t, x):=e^{-r(T-t)} E\left[g\left(X_{T}^{t, x}\right)\right] .
$$

Then, the Malliavin weight representation for the Delta under Black-Scholes model (9) is well-known, that is given by

$$
\frac{\partial}{\partial x} u^{0}(t, x)=e^{-r(T-t)} E\left[g\left(X_{T}^{t, x}\right) \frac{1}{T-t} \int_{t}^{T} \frac{1}{x \sigma} d W_{u}\right] .
$$

In this simple model, we are capable of its evaluation through one dimensional integrations. That is, we have

$$
u^{0}(t, x)=e^{-r(T-t)} \int_{\mathbf{R}} g\left(e^{y}\right) p(t, T, z, y) d y
$$

and

$$
\frac{\partial}{\partial x} u^{0}(t, x)=e^{-r(T-t)} \int_{\mathbf{R}} g\left(e^{y}\right) w(t, z, y) p(t, T, z, y) d y,
$$

where $p(t, T, z, y)$ is the density of $\log X_{T}^{t, x}$ under (9) with $\log x=z$ :

$$
p(t, T, z, y)=\frac{1}{\sqrt{2 \pi \sigma^{2}(T-t)}} \exp \left(-\frac{\left(y-z-\mu(T-t)+\frac{1}{2} \sigma^{2}(T-t)\right)^{2}}{2 \sigma^{2}(T-t)}\right) .
$$

\footnotetext{
${ }^{1}$ The problem is considered under the physical measure and $\left(\frac{\mu-r}{\sigma}\right)$ represents the market price of risk.
} 
the finite dimensional Malliavin weight $w(t, z, y)$ is given by

$$
w(t, z, y)=E\left[\frac{1}{T-t} \int_{t}^{T} \frac{1}{x \sigma} d W_{u} \mid \log X_{T}^{t, x}=y\right]=\frac{\left(y-z-\mu(T-t)+\frac{1}{2} \sigma^{2}(T-t)\right)}{e^{z} \sigma^{2}(T-t)} .
$$

Hence, we get the 0 -th iteration $\left(Y^{0}, Z^{0}\right)=\left(Y_{t}^{0}, Z_{t}^{0}\right)_{t \in[0, T]}$ as

$$
Y_{t}^{0}=u^{0}\left(t, X_{t}\right), \quad Z_{t}^{0}=\sigma X_{t} \frac{\partial}{\partial x} u^{0}\left(t, X_{t}\right)
$$

Next, using the function $u^{0}(t, x)$, we define $u^{1}(t, x)$ as

$$
u^{1}(t, x):=u^{0}(t, x)+e^{-r(T-t)} \int_{t}^{T} \int_{\mathbf{R}} f\left(u^{0}\left(v, e^{y}\right), \sigma e^{y} \frac{\partial}{\partial x} u^{0}\left(v, e^{y}\right)\right) p(t, v, z, y) d y d v,
$$

where $z=\log x$. Then, applying the same weight $w$ as $(16)$, we are able to evaluate $\frac{\partial}{\partial x} u^{1}(t, x)$ :

$$
\begin{aligned}
\frac{\partial}{\partial x} u^{1}(t, x)= & \frac{\partial}{\partial x} u^{0}(t, x) \\
& +e^{-r(T-t)} \int_{t}^{T} \int_{\mathbf{R}} f\left(u^{0}\left(v, e^{y}\right), \sigma e^{y} \frac{\partial}{\partial x} u^{0}\left(v, e^{y}\right)\right) w(v, z, y) p(t, v, z, y) d y d v .
\end{aligned}
$$

Therefore, the first iteration is given by

$$
Y_{t}^{1}=u^{1}\left(t, X_{t}\right), \quad Z_{t}^{1}=\sigma X_{t} \frac{\partial}{\partial x} u^{1}\left(t, X_{t}\right)
$$

Thus, for $k \geq 1$ let us recursively define $u^{k+1}(t, x)$ as

$$
u^{k+1}(t, x):=u^{0}(t, x)+e^{-r(T-t)} \int_{t}^{T} \int_{\mathbf{R}} f\left(u^{k}\left(v, e^{y}\right), \sigma e^{y} \frac{\partial}{\partial x} u^{k}\left(v, e^{y}\right)\right) p(t, v, z, y) d y d v
$$

where $z=\log x$, which leads to the evaluation of $\frac{\partial}{\partial x} u^{k+1}(t, x)$ with the same weight $w$ as $(16)$ :

$$
\begin{aligned}
\frac{\partial}{\partial x} u^{k+1}(t, x)= & \frac{\partial}{\partial x} u^{0}(t, x) \\
& +e^{-r(T-t)} \int_{t}^{T} \int_{\mathbf{R}} f\left(u^{k}\left(v, e^{y}\right), \sigma e^{y} \frac{\partial}{\partial x} u^{k}\left(v, e^{y}\right)\right) w(v, z, y) p(t, v, z, y) d y d v .
\end{aligned}
$$

Hence, the $k+1$-iteration is obtained by

$$
Y_{t}^{k+1}=u^{k+1}\left(t, X_{t}\right), \quad Z_{t}^{k+1}=\sigma X_{t} \frac{\partial}{\partial x} u^{k+1}\left(t, X_{t}\right) .
$$

Finally, applying the parameters so that $X_{0}=100, \sigma=0.2, \mu=0.05, r=0.01, R=0.06, T=0.25$, $K_{1}=95, K_{2}=105$, let us show a numerical comparison of this iterated approximation scheme with their result.

- Benchmark value of $Y_{0}: 2.95$ with 0.01 standard deviation, which is obtained by a regression-based Monte Carlo simulation of Gobet et al. [15].

- Our approximation values: 0 -th iteration $=2.7864$, the first iteration $=2.9671$, and the second iteration $=2.9531$.

It is observed that our approximation values become closer to the benchmark one as the more iterations are implemented. In the following sections, we extend our method in a more general setting.

\section{Summary of algorithm of asymptotic expansion for FBSDEs}

In the example of Section 2, we made use of an explicit Gaussian density since the forward process is given by Black-Scholes model (9). However, when we consider a more complex forward process, the explicit density is no longer obtained in general. Let us consider the perturbed forward SDE (5) with smooth 
coefficients and ellipticity. Then, for $\varepsilon>0$ we are able to derive closed form approximation of the density and its gradient by applying $N$-th order asymptotic expansion around a Gaussian model $\bar{X}_{T}^{t, x}$ :

$$
\begin{aligned}
p^{X^{\varepsilon}}(t, T, x, y) & \simeq p^{\bar{X}}(t, T, x, y)+\sum_{i=1}^{N} \varepsilon^{i} E\left[\pi_{i, T}^{t, x} \mid \bar{X}_{T}^{t, x}=y\right] p^{\bar{X}}(t, T, x, y), \\
\frac{\partial}{\partial x} p^{X^{\varepsilon}}(t, T, x, y) & \simeq E\left[N_{0, T}^{t, x} \mid \bar{X}_{T}^{t, x}=y\right] p^{\bar{X}}(t, T, x, y)+\sum_{i=1}^{N} \varepsilon^{i} E\left[N_{i, T}^{t, x} \mid \bar{X}_{T}^{t, x}=y\right] p^{\bar{X}}(t, T, x, y),
\end{aligned}
$$

with the density $p^{\bar{X}}(t, T, x, y)$ of $\bar{X}_{T}^{t, x}$ and some Malliavin weights $\pi_{i, T}^{t, x}, i=1, \cdots, N$ and $N_{i, T}^{t, x}, i=$ $0,1, \cdots, N$, which are explicitly defined in Section 5 . For the following general BSDE (6) under suitable conditions, we define $\left(u^{\varepsilon}, \partial_{x} u^{\varepsilon} \sigma\right)$ as

$$
\begin{aligned}
u^{\varepsilon}(t, x)= & Y_{t}^{\varepsilon, t, x}=E\left[g\left(X_{T}^{\varepsilon, t, x}\right)\right]+E\left[\int_{t}^{T} f\left(s, X_{s}^{\varepsilon, t, x}, Y_{s}^{\varepsilon, t, x}, Z_{s}^{\varepsilon, t, x}\right) d s\right] \\
= & \int_{\mathbf{R}^{d}} g(y) p^{X^{\varepsilon}}(t, T, x, y) d y+\int_{t}^{T} \int_{\mathbf{R}^{d}} f\left(s, y, u^{\varepsilon}(s, y), \partial_{x} u^{\varepsilon} \sigma(s, y)\right) p^{X^{\varepsilon}}(t, s, x, y) d y d s, \quad(19) \\
\partial_{x} u^{\varepsilon} \sigma(t, x)= & Z_{t}^{\varepsilon, t, x}=E\left[g\left(X_{T}^{\varepsilon, t, x}\right) N_{T}^{\varepsilon, t, x}\right] \varepsilon \sigma(t, x)+E\left[\int_{t}^{T} f\left(s, X_{s}^{\varepsilon, t, x}, Y_{s}^{\varepsilon, t, x}, Z_{s}^{\varepsilon, t, x}\right) N_{s}^{\varepsilon, t, x} d s\right] \varepsilon \sigma(t, x) \\
= & \int_{\mathbf{R}^{d}} g(y) E\left[N_{T}^{\varepsilon, t, x} \mid X_{T}^{\varepsilon, t, x}=y\right] p^{X^{\varepsilon}}(t, T, x, y) d y \varepsilon \sigma(t, x) \\
& \quad+\int_{t}^{T} \int_{\mathbf{R}^{d}} f\left(s, y, u^{\varepsilon}(s, y), \partial_{x} u^{\varepsilon} \sigma(s, y)\right) E\left[N_{s}^{\varepsilon, t, x} \mid X_{s}^{\varepsilon, t, x}=y\right] p^{X^{\varepsilon}}(t, s, x, y) d y d s \varepsilon \sigma(t, x),
\end{aligned}
$$

where $N_{s}^{\varepsilon, t, x}=\frac{1}{\varepsilon(s-t)} \int_{t}^{s} \sigma^{-1}\left(X_{v}^{\varepsilon, t, x}\right) \partial_{x} X_{v}^{\varepsilon, t, x} d W_{v}$. We approximate $\left(u^{\varepsilon}, \partial_{x} u^{\varepsilon} \sigma\right)$ using a sequence $\left(u^{\varepsilon, k, N}, \partial_{x} u^{\varepsilon, k, N} \sigma\right)_{k}$ in the following way.

1. $\left(u^{\varepsilon, 0, N}, \partial_{x} u^{\varepsilon, 0, N} \sigma\right)$ : An approximation of the 0-th iteration

The 0 -th iteration is defined by

$$
\begin{aligned}
u^{\varepsilon, 0}(t, x) & =E\left[g\left(X_{T}^{\varepsilon, t, x}\right)\right]+E\left[\int_{t}^{T} f\left(s, X_{s}^{\varepsilon, t, x}, 0,0\right) d s\right] \\
\partial_{x} u^{\varepsilon, 0} \sigma(t, x) & =E\left[g\left(X_{T}^{\varepsilon, t, x}\right) N_{T}^{\varepsilon, t, x}\right]+E\left[\int_{t}^{T} f\left(s, X_{s}^{\varepsilon, t, x}, 0,0\right) N_{s}^{\varepsilon, t, x} d s\right] .
\end{aligned}
$$

Then, $\left(u^{\varepsilon, 0}, \partial_{x} u^{\varepsilon, 0} \sigma\right)$ is approximated by

$$
\begin{aligned}
Y_{t}^{\varepsilon, t, x}= & u^{\varepsilon, 0}(t, x) \simeq u^{\varepsilon, 0, N}(t, x) \\
= & \int_{\mathbf{R}^{d}} g(y)\left\{1+\sum_{i=1}^{N} \varepsilon^{i} E\left[\pi_{i, T}^{t, x} \mid \bar{X}_{T}^{t, x}=y\right]\right\} p^{\bar{X}}(t, T, x, y) d y \\
& +\int_{t}^{T} \int_{\mathbf{R}^{d}} f(s, y, 0,0)\left\{1+\sum_{i=1}^{N} \varepsilon^{i} E\left[\pi_{i, s}^{t, x} \mid \bar{X}_{s}^{t, x}=y\right]\right\} p^{\bar{X}}(t, s, x, y) d y d s, \\
Z_{t}^{\varepsilon, t, x}= & \partial_{x} u^{\varepsilon, 0} \sigma(t, x) \simeq \partial_{x} u^{\varepsilon, 0, N} \sigma(t, x) \\
= & \int_{\mathbf{R}^{d}} g(y) \sum_{i=0}^{N} \varepsilon^{i} E\left[N_{i, T}^{t, x} \mid \bar{X}_{T}^{t, x}=y\right] p^{\bar{X}}(t, T, x, y) d y \varepsilon \sigma(t, x) \\
& +\int_{t}^{T} \int_{\mathbf{R}^{d}} f(s, y, 0,0) \sum_{0=1}^{N} \varepsilon^{i} E\left[N_{i, s}^{t, x} \mid \bar{X}_{s}^{t, x}=y\right] p^{\bar{X}}(t, s, x, y) d y d s \varepsilon \sigma(t, x) .
\end{aligned}
$$

Note that the Malliavin weights $\pi_{i, s}^{t, x}, i=1, \cdots, N$ and $N_{i, s}^{t, x}, i=0,1, \cdots, N$ are same as in (17) and (18). 
2. $\left(u^{\varepsilon, 1, N}, \partial_{x} u^{\varepsilon, 1, N} \sigma\right)$ : An approximation of the first iteration

The first iteration is defined by

$$
\begin{aligned}
u^{\varepsilon, 1}(t, x) & =E\left[g\left(X_{T}^{\varepsilon, t, x}\right)+\int_{t}^{T} f\left(s, X_{s}^{\varepsilon, t, x}, u^{\varepsilon, 0}\left(s, X_{s}^{\varepsilon, t, x}\right),\left(\partial_{x} u^{\varepsilon, 0} \sigma\right)\left(s, X_{s}^{\varepsilon, t, x}\right)\right) d s\right], \\
\partial_{x} u^{\varepsilon, 1} \sigma(t, x) & =E\left[g\left(X_{T}^{\varepsilon, t, x}\right) N_{T}^{\varepsilon, t, x}+\int_{t}^{T} f\left(s, X_{s}^{\varepsilon, t, x}, u^{\varepsilon, 0}\left(s, X_{s}^{\varepsilon, t, x}\right),\left(\partial_{x} u^{\varepsilon, 0} \sigma\right)\left(s, X_{s}^{\varepsilon, t, x}\right)\right) N_{s}^{\varepsilon, t, x} d s\right] \varepsilon \sigma(t, x) .
\end{aligned}
$$

Firstly, define

$$
\begin{aligned}
\hat{u}^{\varepsilon, 1}(t, x) & =E\left[g\left(X_{T}^{\varepsilon, t, x}\right)+\int_{t}^{T} f\left(s, X_{s}^{\varepsilon, t, x}, u^{\varepsilon, 0, N}\left(s, X_{s}^{\varepsilon, t, x}\right),\left(\partial_{x} u^{\varepsilon, 0, N} \sigma\right)\left(s, X_{s}^{\varepsilon, t, x}\right)\right) d s\right], \\
\partial_{x} \hat{u}^{\varepsilon, 1} \sigma(t, x) & =E\left[g\left(X_{T}^{\varepsilon, t, x}\right)+\int_{t}^{T} f\left(s, X_{s}^{\varepsilon, t, x}, u^{\varepsilon, 0, N}\left(s, X_{s}^{\varepsilon, t, x}\right),\left(\partial_{x} u^{\varepsilon, 0, N} \sigma\right)\left(s, X_{s}^{\varepsilon, t, x}\right)\right) d s\right] \varepsilon \sigma(t, x) .
\end{aligned}
$$

$\left(\hat{u}^{\varepsilon, 1}, \partial_{x} \hat{u}^{\varepsilon, 1} \sigma\right)$ is an approximation of $\left(u^{\varepsilon, 1}, \partial_{x} u^{\varepsilon, 1} \sigma\right)$ :

$$
u^{\varepsilon, 1}(t, x) \simeq \hat{u}^{\varepsilon, 1}(t, x), \quad \partial_{x} u^{\varepsilon, 1} \sigma(t, x) \simeq \partial_{x} \hat{u}^{\varepsilon, 1} \sigma(t, x) .
$$

Using the approximations (17) and (18) again, we expand $\left(\hat{u}^{\varepsilon, 1}, \partial_{x} \hat{u}^{\varepsilon, 1} \sigma\right)$ with respect to $\varepsilon$ as follows:

$$
\begin{aligned}
& \hat{u}^{\varepsilon, 1}(t, x) \\
\simeq \quad & u^{\varepsilon, 1, N}(t, x):=\int_{\mathbf{R}^{d}} g(y)\left\{1+\sum_{i=1}^{N} \varepsilon^{i} E\left[\pi_{i, T}^{t, x} \mid \bar{X}_{T}^{t, x}=y\right]\right\} p^{\bar{X}}(t, T, x, y) d y \\
& +\int_{t}^{T} \int_{\mathbf{R}^{d}} f\left(s, y, u^{\varepsilon, 0, N}(s, y),\left(\partial_{x} u^{\varepsilon, 0, N} \sigma\right)(s, y)\right)\left\{1+\sum_{i=1}^{N} \varepsilon^{i} E\left[\pi_{i, s}^{t, x} \mid \bar{X}_{s}^{t, x}=y\right]\right\} p^{\bar{X}}(t, s, x, y) d y d s . \\
\simeq \quad & \partial_{x} u^{\varepsilon, 1, N} \sigma(t, x):=\int_{\mathbf{R}^{d}} g(y) \sum_{i=0}^{N} \varepsilon^{i} E\left[N_{i, T}^{t, x} \mid \bar{X}_{T}^{t, x}=y\right] p^{\bar{X}}(t, T, x, y) d y \varepsilon \sigma(t, x) \\
& +\int_{t}^{T} \int_{\mathbf{R}^{d}} f\left(s, y, u^{\varepsilon, 0, N}(s, y),\left(\partial_{x} u^{\varepsilon, 0, N} \sigma\right)(s, y)\right) \sum_{i=0}^{N} \varepsilon^{i} E\left[N_{i, s}^{t, x} \mid \bar{X}_{s}^{t, x}=y\right] p^{\bar{X}}(t, s, x, y) d y d s \varepsilon \sigma(t, x) .
\end{aligned}
$$

Since $Y_{t}^{\varepsilon, 1, t, x}=u^{\varepsilon, 1}(t, x)$ and $Z_{t}^{\varepsilon, 1, t, x}=\partial_{x} u^{\varepsilon, 1} \sigma(t, x)$, we get approximation $Y_{t}^{\varepsilon, 1, t, x} \simeq u^{\varepsilon, 1, N}(t, x)$ and $Z_{t}^{\varepsilon, 1, t, x} \simeq \partial_{x} u^{\varepsilon, 1, N_{\sigma}} \sigma(t, x)$ using (25) and (26). Then, $Y_{s}^{\varepsilon, 0, N, t, x}$ and $Z_{s}^{\varepsilon, 0, N, t, x}$ are given by $Y_{s}^{\varepsilon, 0, N, t, x}=u^{\varepsilon, 0, N}\left(s, \bar{X}_{s}^{t, x}\right)$ and $Z_{s}^{\varepsilon, 0, N, t, x}=\partial_{x} u^{\varepsilon, 0, N} \sigma\left(s, \bar{X}_{s}^{t, x}\right)$.

3. Numerical approximation for $\left(u^{\varepsilon}, \partial_{x} u^{\varepsilon} \sigma\right)$

Iterating the procedure above, we obtain the following numerical approximation for $\left(u^{\varepsilon}, \partial_{x} u^{\varepsilon} \sigma\right)$ : for $k \in \mathbf{N}$,

$$
\begin{aligned}
& u^{\varepsilon}(t, x) \\
& \simeq \quad u^{\varepsilon, k, N}(t, x)=\int_{\mathbf{R}^{d}} g(y)\left\{1+\sum_{i=1}^{N} \varepsilon^{i} E\left[\pi_{i, T}^{t, x} \mid \bar{X}_{T}^{t, x}=y\right]\right\} p^{\bar{X}}(t, T, x, y) d y \\
& +\int_{t}^{T} \int_{\mathbf{R}^{d}} f\left(s, y, u^{\varepsilon, k-1, N}(s, y),\left(\partial_{x} u^{\varepsilon, k-1, N} \sigma\right)(s, y)\right) \\
& \left\{1+\sum_{i=1}^{N} \varepsilon^{i} E\left[\pi_{i, s}^{t, x} \mid \bar{X}_{s}^{t, x}=y\right]\right\} p^{\bar{X}}(t, s, x, y) d y d s, \\
& \partial_{x} u^{\varepsilon} \sigma(t, x) \quad \partial_{x} u^{\varepsilon, k, N} \sigma(t, x)=\int_{\mathbf{R}^{d}} g(y) \sum_{i=0}^{N} \varepsilon^{i} E\left[N_{i, T}^{t, x} \mid \bar{X}_{T}^{t, x}=y\right] p^{\bar{X}}(t, T, x, y) d y \varepsilon \sigma(t, x)
\end{aligned}
$$




$$
\begin{array}{r}
+\int_{t}^{T} \int_{\mathbf{R}^{d}} f\left(s, y, u^{\varepsilon, k-1, N}(s, y),\left(\partial_{x} u^{\varepsilon, k-1, N} \sigma\right)(s, y)\right) \\
\sum_{i=0}^{N} \varepsilon^{i} E\left[N_{i, s}^{t, x} \mid \bar{X}_{s}^{t, x}=y\right] p^{\bar{X}}(t, s, x, y) d y d s \varepsilon \sigma(t, x) .
\end{array}
$$

Then, $Y_{s}^{\varepsilon, k, N, t, x}$ and $Z_{s}^{\varepsilon, k, N, t, x}$ are given by $Y_{s}^{\varepsilon, k, N, t, x}=u^{\varepsilon, k, N}\left(s, \bar{X}_{s}^{t, x}\right)$ and $Z_{s}^{\varepsilon, k, N, t, x}=\partial_{x} u^{\varepsilon, k, N} \sigma\left(s, \bar{X}_{s}^{t, x}\right)$.

We show this conjecture and derivation rigorously using Malliavin calculus in Section 5.

\section{Notations and basic results}

Hereafter, we use the following notations. Let $\mathbf{E}$ (or $\mathbf{E}_{1}$ ) be a generic Euclidean space.

- $\partial_{x}: \partial_{x}=\left(\frac{\partial}{\partial x_{1}}, \cdots, \frac{\partial}{\partial x_{d}}\right)$.

- $C(T, x)$ : a generic non-negative, non-decreasing and finite function of at most polynomial growth in $x$ depending on $T>0$.

- $C_{b}^{\infty}\left(\mathbf{E} ; \mathbf{E}_{1}\right)$ : the space of all infinitely differentiable functions $\varphi: \mathbf{E} \rightarrow \mathbf{E}_{1}$ such that the all of its derivatives are bounded. We write $C_{b}^{\infty}(\mathbf{E})$ for $C_{b}^{\infty}(\mathbf{E} ; \mathbf{R})$.

We also prepare the basic notations and definitions of Malliavin calculus.

- $(\Omega, H, P)$ : the standard $d$-dimensional Wiener space.

$-\Omega:$ the continuous functions $w:[0, T] \rightarrow \mathbf{R}^{d}$ such that $w(0)=0$.

- $H$ : the Cameron-Martin space of all absolutely continuous functions $h:[0, T] \rightarrow \mathbf{R}^{d}$ with a square integrable derivative, i.e., $h^{\prime} \in L^{2}\left([0, T] ; \mathbf{R}^{d}\right), h^{\prime}(t)=\frac{d}{d t} h(t)$. Here, $L^{2}\left([0, T] ; \mathbf{R}^{d}\right)$ is the space of all $\mathbf{R}^{d}$-measurable functions $\varphi$ on $[0, T]$ such that $\left(\int_{0}^{T}|\varphi(s)|^{2} d s\right)^{1 / 2}<\infty$.

- $P$ : the Wiener measure.

- $L^{p}(\Omega ; G)$ : the space of all random variables $F: \Omega \rightarrow G$ such that $E\left[\|F\|_{G}^{p}\right]<\infty$ where $G$ is a separable Hilbert space equipped with the norm $\|\cdot\|_{G}$ and $p \in[1, \infty)$. We write $L^{p}(\Omega)$ when $G=\mathbf{R}$ and $\|F\|_{p}=E\left[|F|^{p}\right]^{1 / p}$ for $F \in L^{p}(\Omega)$.

- $\mathcal{S}$ : The set of random variables $F$ of the form

$$
F=\varphi\left(\int_{0}^{T} h_{1}^{\prime}(s) d W_{s}, \cdots, \int_{0}^{T} h_{n}^{\prime}(s) d W_{s}\right)
$$

where $\varphi \in C_{b}^{\infty}\left(\mathbf{R}^{d}\right), h_{1}, \cdots, h_{n} \in H, n \geq 1$ with the notation $\int_{0}^{T} h_{i}^{\prime}(s) d W_{s}=\sum_{j=1}^{d} \int_{0}^{T} h_{i, j}^{\prime}(s) d W_{s}^{i}$, $h_{i}^{\prime}=\left(h_{i, 1}^{\prime}, \cdots, h_{i, d}^{\prime}\right)$.

- Malliavin derivative operator $D$ : If $F \in \mathcal{S}$ is of the above form, we define its derivative as follows

$$
D F=\sum_{i=1}^{n} \frac{\partial \varphi}{\partial x^{i}}\left(\int_{0}^{T} h_{1}^{\prime}(s) d W_{s}, \cdots, \int_{0}^{T} h_{n}^{\prime}(s) d W_{s}\right) h_{i} .
$$

The operator $D$ is closable from $L^{p}(\Omega)$ to $L^{p}(\Omega ; H)$ for any $p \geq 1$.

- $\mathbf{D}^{k, p}$ : For $F \in \mathcal{S}$, the iterated derivative $D^{j} F, j \in \mathbf{N}$ as a random variable with values in $H^{\otimes j}$. We denote by $\mathbf{D}^{k, p}$ the closure of $\mathcal{S}$ with respect to the seminorm

$$
\|F\|_{k, p}=\left(E\left[|F|^{p}\right]+\sum_{j=1}^{k} E\left[\left\|D^{j} F\right\|_{H \otimes j}^{p}\right]\right)^{1 / p}, p \in[1, \infty), k \in \mathbf{N} .
$$

Since $D^{j}$ is closable from $L^{p}(\Omega)$ to $L^{p}\left(\Omega ; H^{\otimes j}\right)$ for any $p \in[1, \infty)$ and $j \in \mathbf{N}, D^{j}$ is well defined in $\mathbf{D}^{k, p}$.

- $\mathbf{D}^{\infty}: \mathbf{D}^{\infty}=\cap_{p \geq 1} \cap_{k \geq 1} \mathbf{D}^{k, p}$. 
- Skorohod integral $\delta$ : We denote by $\delta$ the adjoint operator of the derivative operator $D$, that is an unbounded operator from $L^{2}(\Omega ; H)$ into $L^{2}(\Omega)$ such that the domain of $\delta$, denoted by $\operatorname{Dom}(\delta)$, is the set of $H$-valued square integrable random variables $u$ such that $\left|E\left[\langle D F, u\rangle_{H}\right]\right| \leq C\|F\|_{2}$, for all $F \in \mathbf{D}^{1,2}$, where $C$ is some constant depending on $u$. For $u \in \operatorname{Dom}(\delta), \delta(u)$ is characterized by the duality relationship:

$$
E[F \delta(u)]=E\left[\langle D F, u\rangle_{H}\right], \text { for any } F \in \mathbf{D}^{1,2} .
$$

$\delta(u)$ is called Skorohod integral of the process $u$.

- $\mathbf{D}^{-\infty}$ : the space of the Watanabe distributions (the dual of $\mathbf{D}^{\infty}$ ).

We say $F^{\varepsilon}=O\left(\varepsilon^{n}\right)$ in $\mathbf{D}^{k, p}$ as $\varepsilon \downarrow 0$ if $F^{\varepsilon} \in \mathbf{D}^{k, p}$ for all $\varepsilon \in(0,1]$ and $\lim \sup _{\varepsilon \downarrow 0}\left\|F^{\varepsilon}\right\|_{\mathbf{D}^{k, p}} / \varepsilon^{n}<\infty$ where $n$ is some real constant.

In our algorithm summarized in Section 3, we need to compute the asymptotic expansion $u^{k, N}$ recursively. From a numerical viewpoint, the stability of the approximation i.e. the asymptotic behavior of the asymptotic expansion when $t \uparrow T$ must be checked since we iteratively integrate $u^{k, N}$ with respect to time $t$. Hence, we introduce the Kusuoka-Stroock functions (Kusuoka [18]) which help to clarify the order of a Wiener functional with respect to time $t$.

Definition 4.1 (Kusuoka-Stroock functions) Given $r \in \mathbf{R}$ and $n \in \mathbf{N}$, we denote by $\mathcal{K}_{r}^{T}(n)$ the set of functions $G:(0, T] \times \mathbf{R}^{d} \rightarrow \mathbf{D}^{n, \infty}$ satisfying the followings:

1. $G(t, \cdot)$ is n-times continuously differentiable and $\left[\partial^{\alpha} G / \partial x^{\alpha}\right]$ is continuous in $(t, x) \in(0, T] \times \mathbf{R}^{d}$ a.s. for any multi-index $\alpha$ of the elements of $\{1, \cdots, d\}$ with length $|\alpha| \leq n$.

2. For all $k \leq n-|\alpha|, p \in[1, \infty)$,

$$
\sup _{t \in(0, T], x \in \mathbf{R}^{d}} t^{-r / 2}\left\|\frac{\partial^{\alpha} G}{\partial x^{\alpha}}(t, x)\right\|_{k, p}<\infty .
$$

The above definition corresponds to Definition 2.1 of Crisan and Delarue [5] of modified version of Kusuoka [18]. We write $\mathcal{K}_{r}^{T}$ for $\cap_{n \in \mathbf{N}} \mathcal{K}_{r}^{T}(n)$.

Let $\left(X_{s}^{t, x}\right)$ be the solution to the following stochastic differential equation:

$$
\begin{aligned}
X_{s}^{t, x} & =x+\int_{t}^{s} V_{0}\left(X_{u}^{t, x}\right) d u+\sum_{i=1}^{d} \int_{t}^{s} V_{i}\left(X_{u}^{t, x}\right) d W_{u}^{i}, \\
X_{t}^{t, x} & =x \in \mathbf{R}^{d},
\end{aligned}
$$

where each $V_{i}, i=0,1, \cdots, d$ is bounded and belongs to $C_{b}^{\infty}\left(\mathbf{R}^{d} ; \mathbf{R}^{d}\right)$. We assume that the elliptic condition holds.

Lemma 4.1 [Properties of Kusuoka-Stroock functions] The followings hold.

1. The function $(s, x) \in(0, T] \times \mathbf{R}^{d} \mapsto X_{s}^{t, x}$ belongs to $\mathcal{K}_{0}^{T}$, for any $T>0$.

2. Suppose $G \in \mathcal{K}_{r}^{T}(n)$ where $r \geq 0$. Then, for $i=1, \cdots, d$,

$$
\int_{0}^{.} G(s, x) d W_{s}^{i} \in \mathcal{K}_{r+1}^{T}(n) \text { and } \int_{0}^{.} G(s, x) d s \in \mathcal{K}_{r+2}^{T}(n) .
$$

3. If $G_{i} \in \mathcal{K}_{r_{i}}^{T}\left(n_{i}\right), i=1, \cdots, N$, then

$$
\prod_{i}^{N} G_{i} \in \mathcal{K}_{r_{1}+\cdots+r_{N}}^{T}\left(\min _{i} n_{i}\right) \text { and } \sum_{i=1}^{N} G_{i} \in \mathcal{K}_{\min _{i} r_{i}}^{T}\left(\min _{i} n_{i}\right) .
$$

Proof. See Lemma 5.1.2 of Nee [26] for instance.

Next, we summarize the Malliavin's integration by parts formula using Kusuoka-Stroock functions. For any multi-index $\alpha^{(k)}:=\left(\alpha_{1}, \cdots, \alpha_{k}\right) \in\{1, \cdots, d\}^{k}, k \geq 1$, we denote by $\partial_{\alpha^{(k)}}$ the partial derivative $\frac{\partial^{k}}{\partial x_{\alpha_{1}} \cdots \partial x_{\alpha_{k}}}$. 
Proposition 4.1 Let $G:(0, T] \times \mathbf{R}^{d} \rightarrow \mathbf{D}^{\infty}=\mathbf{D}^{\infty, \infty}$ be an element of $\mathcal{K}_{r}^{T}$ and let $f$ be a function that belongs to the space $C_{b}^{\infty}\left(\mathbf{R}^{d}\right)$. Then for any multi-index $\alpha^{(k)} \in\{1, \cdots, d\}^{k}, k \geq 1$, there exists $H_{\alpha^{(k)}}\left(X_{s}^{t, x}, G(s, x)\right) \in \mathcal{K}_{r-k}^{T}$ such that

$$
E\left[\partial_{\alpha^{(k)}} f\left(X_{s}^{t, x}\right) G(s, x)\right]=E\left[f\left(X_{s}^{t, x}\right) H_{\alpha^{(k)}}\left(X_{s}^{t, x}, G(s, x)\right)\right]
$$

with

$$
\sup _{x \in \mathbf{R}^{d}}\left\|H_{\alpha^{(k)}}\left(X_{s}^{t, x}, G(s, x)\right)\right\|_{p} \leq C(s-t)^{(r-k) / 2},
$$

where $H_{\alpha^{(k)}}\left(X_{s}^{t, x}, G(s, x)\right)$ is recursively given by

$$
\begin{aligned}
H_{(i)}\left(X_{s}^{t, x}, G(s, x)\right) & =\delta\left(\sum_{j=1}^{d} G(s, x) \gamma_{i j}^{X_{s}^{t, x}} D X_{s}^{t, x, j}\right), \\
H_{\alpha^{(k)}}\left(X_{s}^{t, x}, G(s, x)\right) & =H_{\left(\alpha_{k}\right)}\left(X_{s}^{t, x}, H_{\alpha^{(k-1)}}\left(X_{s}^{t, x}, G(s, x)\right)\right),
\end{aligned}
$$

and a positive constant $C$. Here, $\left(\gamma_{i j}^{X_{s}^{t, x}}\right)_{1 \leq i, j \leq d}$ is the inverse matrix of the Malliavin covariance of $X_{s}^{t, x}$. Proof. Apply Corollary 3.7 of Kusuoka and Stroock [19] and Lemma 8-(3) of Kusuoka [18] with Proposition 2.1.4 of Nualart [25].

\section{Asymptotic expansion for FBSDEs}

\subsection{Forward-backward SDE}

Let $(\Omega, H, P)$ be the Wiener space on which a $d$-dimensional Brownian motion $W=\left(W^{1}, \cdots, W^{d}\right)$ is defined. Let $\mathcal{F}$ be the Borel algebra over $\Omega$ and $\left(\mathcal{F}_{t}\right)_{t \geq 0}$ be the natural filtration generated by $W$, augmented by the $P$-null sets of $\mathcal{F}$. In this section, we deal with a small diffusion expansion which corresponds to the framework in Kunitomo and Takahashi [16][17] and derive a general approximation formula for FBSDEs.

We give precise framework of our model. Consider the following $d$-dimensional perturbed forward stochastic differential equation $X_{t}^{\varepsilon}=\left(X_{t}^{1, \varepsilon}, \cdots, X_{t}^{d, \varepsilon}\right)$ :

$$
d X_{t}^{i, \varepsilon}=b^{i}\left(t, X_{t}^{\varepsilon}\right) d t+\varepsilon \sum_{j=1}^{d} \sigma_{j}^{i}\left(t, X_{t}^{\varepsilon}\right) d W_{t}^{j}, \quad i=1, \cdots, d,
$$

where $b:[0, T] \times \mathbf{R}^{d} \rightarrow \mathbf{R}^{d}, \sigma:[0, T] \times \mathbf{R}^{d} \rightarrow \mathbf{R}^{d \times d}$ and $\varepsilon \in(0,1]$.

Next, we introduce the associated $\operatorname{BSDE}\left(Y^{\varepsilon}, Z^{\varepsilon}\right)$ as follows:

$$
Y_{t}^{\varepsilon}=g\left(X_{T}^{\varepsilon}\right)+\int_{t}^{T} f\left(s, X_{s}^{\varepsilon}, Y_{s}^{\varepsilon}, Z_{s}^{\varepsilon}\right) d s-\int_{t}^{T} Z_{s}^{\varepsilon} d W_{s},
$$

where $g: \mathbf{R}^{d} \rightarrow \mathbf{R}$ and $f:[0, T] \times \mathbf{R}^{d} \times \mathbf{R} \times \mathbf{R}^{d} \rightarrow \mathbf{R}$. Remark that for $\varepsilon=0$, since the forward SDE $X_{t}^{0}$ degenerates, does $\operatorname{BSDE}\left(Y_{t}^{0}, Z_{t}^{0}\right)$, too.

We put some conditions below on the above forward-backward SDE.

\section{Assumption 5.1}

1. The coefficients of forward process $b, \sigma$ are bounded Borel functions and $C_{b}^{\infty}$ in $x$.

2. There exist constants $a_{i}>0, i=1,2$ such that for any vector $\xi$ in $\mathbf{R}^{d}$ and any $(t, x) \in[0, T] \times \mathbf{R}^{d}$,

$$
a_{1}|\xi|^{2} \leq \sum_{i, j=1}^{d}\left[\sigma \sigma^{\top}\right]_{i, j}(t, x) \xi_{i} \xi_{j} \leq a_{2}|\xi|^{2}
$$

3. The driver $f:[0, T] \times \mathbf{R}^{d} \times \mathbf{R} \times \mathbf{R}^{d} \rightarrow \mathbf{R}$ is continuous in $t$ and uniformly Lipschitz continuous in $x, y, z$ with constant $C_{L}$, i.e. for all $t \in[0, T],\left(x_{1}, y_{1}, z_{1}\right),\left(x_{2}, y_{2}, z_{2}\right) \in \mathbf{R}^{d} \times \mathbf{R} \times \mathbf{R}^{d}$,

$$
\left|f\left(t, x_{1}, y_{1}, z_{1}\right)-f\left(t, x_{2}, y_{2}, z_{2}\right)\right| \leq C_{L}\left(\left|x_{1}-x_{2}\right|+\left|y_{1}-y_{2}\right|+\left|z_{1}-z_{2}\right|\right) \text {. }
$$

Also, we assume

$$
|f(t, x, y, z)| \leq C_{L}(1+|x|+|y|+|z|) .
$$

for $(t, x, y, z) \in[0, T] \times \mathbf{R}^{d} \times \mathbf{R} \times \mathbf{R}^{d}$. 
4. $g$ is Lipschitz continuous function with constant $C_{L}$ on $\mathbf{R}^{d}$ and $|g(x)| \leq C_{L}(1+|x|)$ for $x \in \mathbf{R}^{d}$.

Under the assumption, there exists the unique solution $\left(Y^{\varepsilon}, Z^{\varepsilon}\right)$ such that for any $p>1, E\left[\sup _{0 \leq s \leq T}\left|Y_{s}^{\varepsilon}\right|^{p}\right]+$ $E\left[\left(\int_{0}^{T}\left|Z_{s}^{\varepsilon}\right|^{2} d s\right)^{p / 2}\right]<\infty$ (e.g. See Theorem 5.1 in [7]).

$\left(X^{\varepsilon, t, x}, Y^{\varepsilon, t, x}, Z^{\varepsilon, t, x}\right)$ represents the adapted solutions to the FBSDE's (37) and (38), restricted to $[t, T]$ with $X_{t}^{\varepsilon, t, x}=x$, a.s. The representation (20) of Ma and Zhang [23] holds under Assumption 5.1.

\section{$5.2 \quad$ Asymptotic expansion}

Under 1 and 2 in Assumption 5.1, the solution to SDE $X_{s}^{\varepsilon, t, x}(0 \leq t<s \leq T)$ has a smooth density $p^{X^{\varepsilon}}(t, s, x, y)$. In order to obtain the expansion of the density $p^{X^{\varepsilon}}(t, s, x, y)$, we approximate $X_{s}^{\varepsilon, t, x}$ by an asymptotic expansion around the solution to ordinary differential equation $X_{s}^{0, t, x}=x+\int_{t}^{s} b\left(u, X_{u}^{0, t, x}\right) d s$.

Hereafter, let us denote by $X_{i, s}^{\varepsilon, t, x}, i \in \mathbf{N}$ the $i$-th order differentiation of $X_{s}^{\varepsilon, t, x}$ with respect to $\varepsilon$, i.e. $\frac{1}{i !} \frac{\partial^{i}}{\partial \varepsilon^{i}} X_{s}^{\varepsilon, t, x}$. In the first place, we provide a key result as the lemma below.

Lemma 5.1 For $s \in(t, T]$, we have $X_{i, s}^{\varepsilon, t, x} \in \mathcal{K}_{i}^{T}, i \in \mathbf{N}$.

Proof. See Appendix A.

Let us define $X_{i, s}^{0, t, x}$ as $\left.\frac{1}{i !} \frac{\partial^{i}}{\partial \varepsilon^{i}} X_{s}^{\varepsilon, t, x}\right|_{\varepsilon=0}, i \in \mathbf{N}$. For every $p \in[1, \infty), k \in \mathbf{N}$ and $N \in \mathbf{N}$,

$$
X_{s}^{\varepsilon, t, x}=X_{s}^{0, t, x}+\sum_{i=1}^{N} \varepsilon^{i} X_{i, s}^{0, t, x}+O\left(\varepsilon^{N+1}\right) \text { in } \mathbf{D}^{k, p} \text { as } \varepsilon \downarrow 0 .
$$

Hereafter, we derive an asymptotic expansion of density of $X_{T}^{\varepsilon, t, x}$. Define $F_{T}^{\varepsilon, t, x}$ as $F_{T}^{\varepsilon, t, x}:=\frac{X_{T}^{\varepsilon, t, x}-X_{T}^{0, t, x}}{\varepsilon}$ and then we have

$$
F_{T}^{\varepsilon, t, x}=F_{T}^{0, t, x}+\sum_{i=1}^{N} \varepsilon^{i} F_{i, T}^{0, t, x}+O\left(\varepsilon^{N+1}\right) \text { in } \mathbf{D}^{\infty},
$$

where $F_{T}^{0, t, x}=X_{1, T}^{0, t, x}, F_{i, T}^{0, t, x}=X_{i+1, T}^{0, t, x}, i \geq 1$.

Let $\Sigma(t, T)=\left\{\Sigma_{i, j}(t, T)\right\}_{i, j}$ be the $d \times d$-matrix whose element is defined by

$$
\Sigma_{i, j}(t, T)=\sum_{k=1}^{d} \int_{t}^{T} \hat{\sigma}_{k}^{i}\left(s, X_{s}^{0, t, x}\right) \hat{\sigma}_{k}^{j}\left(s, X_{s}^{0, t, x}\right) d s, \quad 1 \leq i, j \leq d,
$$

where $\hat{\sigma}_{k}^{i}\left(s, X_{s}^{0, t, x}\right)=\left(\partial_{x} X_{T}^{0, t, x}\left(\partial_{x} X_{s}^{0, t, x}\right)^{-1} \sigma_{k}\left(s, X_{s}^{0, t, x}\right)\right)^{i}$.

Under Assumption 5.1 we obtain the following expansions for $E\left[\varphi\left(X_{T}^{\varepsilon, t, x}\right)\right]$ with Lipschitz function $\varphi$, which are useful for giving the properties of the expansion of $Y^{\varepsilon}$ and proving our main result Theorem 5.1 .

Proposition 5.1 For $N \in \mathbf{N}$ and a Lipschitz continuous function $\varphi: \mathbf{R}^{d} \rightarrow \mathbf{R}$ with constant $C_{L}$, there exists $C_{N}$ depending on $C_{L}$ and $N$ such that

$$
\left|E\left[\varphi\left(X_{T}^{\varepsilon, t, x}\right)\right]-\left\{E\left[\varphi\left(\bar{X}_{T}^{t, x}\right)\right]+\sum_{i=1}^{N} \varepsilon^{i} E\left[\varphi\left(\bar{X}_{T}^{t, x}\right) \pi_{i, T}^{t, x}\right]\right\}\right| \leq \varepsilon^{N+1} C_{N}(T-t)^{(N+2) / 2},
$$

where $\bar{X}_{T}^{t, x}=X_{T}^{0, t, x}+\varepsilon X_{1, T}^{0, t, x}$ and $\pi_{i, T}^{t, x}=\sum_{k}^{(i)} H_{\alpha^{(k)}}\left(X_{1, T}^{0, t, x}, \prod_{l=1}^{k} X_{\beta_{l}+1, T}^{0, t, x, \alpha_{l}}\right) \in \mathcal{K}_{i}^{T}, i=1, \cdots, N$. Here, $\sum_{k}^{(i)} \equiv \sum_{k=1}^{i} \sum_{\beta_{1}+\cdots+\beta_{k}=i, \beta_{j} \geq 1} \sum_{\alpha^{(k)} \in\{1, \cdots, d\}^{k}} \frac{1}{k !}$.

Proof. See Appendix B.

We also obtain an expansion for $E\left[\varphi\left(X_{T}^{\varepsilon, t, x}\right) N_{T}^{\varepsilon, t, x}\right] \varepsilon \sigma(t, x)$ with Lipschitz function $\varphi$, which are useful for giving the properties of the expansion of $Z^{\varepsilon}$. 
Proposition 5.2 For $N \in \mathbf{N}$ and a Lipschitz continuous function $\varphi: \mathbf{R}^{d} \rightarrow \mathbf{R}$ with constant $C_{L}$, there exists $C_{N}$ depending on $C_{L}$ and $N$ such that

$$
\begin{aligned}
& \left|E\left[\varphi\left(X_{T}^{\varepsilon, t, x}\right) N_{0, T}^{\varepsilon, t, x}\right] \varepsilon \sigma(t, x)-\left\{E\left[\varphi\left(\bar{X}_{T}^{t, x}\right) N_{0, T}^{t, x}\right]+\sum_{i=1}^{N} \varepsilon^{i} E\left[\varphi\left(\bar{X}_{T}^{t, x}\right) N_{i, T}^{t, x}\right]\right\} \varepsilon \sigma(t, x)\right| \\
& \leq \varepsilon^{N+1} C_{N}(T-t)^{(N+1) / 2},
\end{aligned}
$$

where $\bar{X}_{T}^{t, x}=X_{T}^{0, t, x}+\varepsilon X_{1, T}^{0, t, x}, N_{0, T}^{t, x}=\left(N_{0, T}^{t, x, 1} \cdots, N_{0, T}^{t, x, d}\right)$ and $N_{i, T}^{t, x}=\left(N_{i, T}^{t, x, 1}, \cdots, N_{i, T}^{t, x, d}\right), i=1, \cdots, d$ are given by $N_{0, T}^{t, x, \eta}=\sum_{j=1}^{d} H_{(j)}\left(\bar{X}_{T}^{t, x}, \partial_{\eta} \bar{X}_{T}^{t, x, j}\right)$, and $N_{i, T}^{t, x, \eta}=\sum_{j=1}^{d} H_{(j)}\left(\bar{X}_{T}^{t, x}, \partial_{\eta} \bar{X}_{T}^{t, x, j} \pi_{i, T}^{t, x}\right)+\partial_{\eta} \pi_{i, T}^{t, x}$, $1 \leq \eta \leq d$

Proof. See Appendix C.

Remark 5.1 Using the similar arguments in Proposition 5.1 and 5.2, we are able to see the following results. For a measurable function $\varphi: \mathbf{R}^{d} \rightarrow \mathbf{R}$ of at most polynomial growth, there exists non-negative, non-decreasing and finite function $C(N, x)$ of at most polynomial growth in $x$ depending on $N$ such that

$$
\begin{aligned}
& \left|E\left[\varphi\left(X_{T}^{\varepsilon, t, x}\right)\right]-\left\{E\left[\varphi\left(\bar{X}_{T}^{t, x}\right)\right]+\sum_{i=1}^{N} \varepsilon^{i} E\left[\varphi\left(\bar{X}_{T}^{t, x}\right) \pi_{i, T}^{t, x}\right]\right\}\right| \leq \varepsilon^{N+1} C(N, x)(T-t)^{(N+1) / 2}, \\
& \left|E\left[\varphi\left(X_{T}^{\varepsilon, t, x}\right) N_{0, T}^{\varepsilon, t, x}\right] \varepsilon \sigma(t, x)-\left\{E\left[\varphi\left(\bar{X}_{T}^{t, x}\right) N_{0, T}^{t, x}\right]+\sum_{i=1}^{N} \varepsilon^{i} E\left[\varphi\left(\bar{X}_{T}^{t, x}\right) N_{i, T}^{t, x}\right]\right\} \varepsilon \sigma(t, x)\right| \\
\leq & \varepsilon^{N+1} C(N, x)(T-t)^{N / 2},
\end{aligned}
$$

with the same weights in Proposition 5.1 and 5.2. In the above estimates, we do not use the smoothness of $\varphi$ while we use the Lipschitz differentiability in Proposition 5.1 and 5.2.

Using the weights $\pi_{i, s}^{t, x}, i=0,1, \cdots, N$ in Proposition 5.1 and $N_{i, s}^{t, x}, i=0,1, \cdots, N$ in Proposition 5.2, we have formulas for $\left(u^{\varepsilon, k, N}, \partial_{x} u^{\varepsilon, k, N} \sigma\right)$ as (27) and (28) without using derivatives of $f$ and $g$.

The following property holds for $\left(u^{\varepsilon, k, N}, \partial_{x} u^{\varepsilon, k, N} \sigma\right)$ by Lipschitz continuity of $g$.

Lemma 5.2 For $k \geq 0, N \in \mathbf{N}$,

$$
\begin{aligned}
\left|u^{\varepsilon, k, N}(t, x)\right| & \leq C(T, x), \\
\left|\partial_{x} u^{\varepsilon, k, N} \sigma(t, x)\right| & \leq C(T, x) .
\end{aligned}
$$

where $C(T, x)$ denotes a generic non-negative, non-decreasing and finite function of at most polynomial growth in $x$ depending on $T$.

Proof. See Appendix D.

\subsection{Error estimate}

For any $\beta, \mu>0$, let $H_{\beta, \mu}$ be the space of functions $v:[0, T] \times \mathbf{R}^{d} \rightarrow \mathbf{R}^{n}$ such that

$$
\|v\|_{H_{\beta, \mu}}^{2}=\int_{0}^{T} \int_{\mathbf{R}^{d}} e^{\beta s}|v(s, x)|^{2} e^{-\mu|x|} d x d s<\infty .
$$

We also define the space $H_{\beta, \mu, X}$, For any $\beta, \mu>0$, and $X_{s}^{\varepsilon}, 0 \leq s \leq T$ starting from $x$ at time 0 , let $H_{\beta, \mu, X}$ be the space of functions $v:[0, T] \times \mathbf{R}^{d} \rightarrow \mathbf{R}^{n}$ such that

$$
\|v\|_{H_{\beta, \mu, X}}^{2}=\int_{0}^{T} \int_{\mathbf{R}^{d}} e^{\beta s} E\left[\left|v\left(s, X_{s}^{\varepsilon}\right)\right|^{2}\right] e^{-\mu|x|} d x d s<\infty .
$$

Remark that the following well-known norm equivalence result holds (e.g. Proposition 3.8 in Gobet and Labert [14]): there exist two constants $c_{1}, c_{2}>0$ such that $v \in L^{2}\left([0, T] \times \mathbf{R}^{d}, e^{\beta s} d s \times e^{-\mu|x|} d x\right)$

$$
c_{1}\|v\|_{H_{\beta, \mu}}^{2} \leq\|v\|_{H_{\beta, \mu, X}}^{2} \leq c_{2}\|v\|_{H_{\beta, \mu}}^{2} .
$$


The next theorem is our main result, which evaluates a global approximation error of $\left(u^{\varepsilon, k, N}, \partial_{x} u^{\varepsilon, k, N} \sigma\right)$ (in $(27)$ and (28)) for $\left(u^{\varepsilon}, \partial_{x} u^{\varepsilon} \sigma\right)$ (in (19) and (20)).

Theorem 5.1 Suppose that Assumption 5.1 holds. Let $C$ be $C=c_{2} / c_{1}$ and $\beta$ be such that $2 C C_{L}^{2}(T+1)<$ $\beta$ and fix $\delta:=\frac{2 C C_{L}^{2}(T+1)}{\beta}<1$. Then, for arbitrary $k \geq 0$ and $N \in \mathbf{N}$, there exists $C_{0}(T)$ depending on $T$ and $C_{1}(T, N)$ depending on $T$ and $N$ such that

$$
\begin{aligned}
& \left\|u^{\varepsilon}-u^{\varepsilon, k, N}\right\|_{H_{\beta, \mu}}^{2}+\left\|\left(\partial_{x} u^{\varepsilon} \sigma\right)-\left(\partial_{x} u^{\varepsilon, k, N} \sigma\right)\right\|_{H_{\beta, \mu}}^{2} \\
& \quad \leq\left\{C_{0}(T) \cdot \delta^{k}+\varepsilon^{2(N+1)} C_{1}(T, N) \cdot\left(\frac{1-\delta^{k+1}}{1-\delta}\right)\right\}, \quad \varepsilon \in(0,1] .
\end{aligned}
$$

\section{Proof.}

Note that the following inequality holds:

$$
\begin{aligned}
& \left\|u^{\varepsilon}-u^{\varepsilon, k, N}\right\|_{H_{\beta, \mu}}^{2}+\left\|\partial_{x} u^{\varepsilon} \sigma-\partial_{x} u^{\varepsilon, k, N} \sigma\right\|_{H_{\beta, \mu}}^{2} \\
\leq & 2\left(\left\|u^{\varepsilon}-u^{\varepsilon, k}\right\|_{H_{\beta, \mu}}^{2}+\left\|\partial_{x} u^{\varepsilon} \sigma-\partial_{x} u^{\varepsilon, k} \sigma\right\|_{H_{\beta, \mu}}^{2}\right) \\
& +2\left(\left\|u^{\varepsilon, k}-u^{\varepsilon, k, N}\right\|_{H_{\beta, \mu}}^{2}+\left\|\partial_{x} u^{\varepsilon, k} \sigma-\partial_{x} u^{\varepsilon, k, N} \sigma\right\|_{H_{\beta, \mu}}^{2}\right) .
\end{aligned}
$$

First, we show the error $\left\|u^{\varepsilon}-u^{\varepsilon, k}\right\|_{H_{\beta, \mu}}^{2}+\left\|\left(\partial_{x} u^{\varepsilon} \sigma\right)-\left(\partial_{x} u^{\varepsilon, k} \sigma\right)\right\|_{H_{\beta, \mu}}^{2}$ by using the norm equivalence, (51) and the similar argument in the proof of Theorem 2.1 in El Karoui et al. [7]:

$$
\begin{aligned}
& \left\|u^{\varepsilon}-u^{\varepsilon, k}\right\|_{H_{\beta, \mu}}^{2}+\left\|\left(\partial_{x} u^{\varepsilon} \sigma\right)-\left(\partial_{x} u^{\varepsilon, k} \sigma\right)\right\|_{H_{\beta, \mu}}^{2} \\
\leq & \frac{2 C C_{L}^{2}(T+1)}{\beta}\left\{\left\|u^{\varepsilon}-u^{\varepsilon, k-1}\right\|_{H_{\beta, \mu}}^{2}+\left\|\left(\partial_{x} u^{\varepsilon} \sigma\right)-\left(\partial_{x} u^{\varepsilon, k-1} \sigma\right)\right\|_{H_{\beta, \mu}}^{2}\right\} .
\end{aligned}
$$

Therefore,

$$
\left\|u^{\varepsilon}-u^{\varepsilon, k}\right\|_{H_{\beta, \mu}}^{2}+\left\|\left(\partial_{x} u^{\varepsilon} \sigma\right)-\left(\partial_{x} u^{\varepsilon, k} \sigma\right)\right\|_{H_{\beta, \mu}}^{2} \leq C_{0}(T) \cdot\left(\frac{2 C C_{L}^{2}(T+1)}{\beta}\right)^{k},
$$

where $C_{0}(T)$ such that $\left\|u^{\varepsilon}-u^{\varepsilon, 0}\right\|_{H_{\beta, \mu}}^{2}+\left\|\left(\partial_{x} u^{\varepsilon} \sigma\right)-\left(\partial_{x} u^{\varepsilon, 0} \sigma\right)\right\|_{H_{\beta, \mu}}^{2} \leq C_{0}(T)$.

Next, we estimate the error $\left\|u^{\varepsilon, k}-u^{\varepsilon, k, N}\right\|_{H_{\beta, \mu}}^{2}+\left\|\partial_{x} u^{\varepsilon, k} \sigma-\partial_{x} u^{\varepsilon, k, N} \sigma\right\|_{H_{\beta, \mu}}^{2}$.

The difference $u^{\varepsilon, k+1}-u^{\varepsilon, k+1, N}$ is represented as follows:

$$
\begin{aligned}
& u^{\varepsilon, k+1}(t, x)-u^{\varepsilon, k+1, N}(t, x) \\
= & \int_{\mathbf{R}^{d}} g(y) p^{X^{\varepsilon}}(t, T, x, y) d y+\int_{t}^{T} \int_{\mathbf{R}^{d}} f\left(s, y, u^{\varepsilon, k}(s, y),\left(\partial_{x} u^{\varepsilon, k} \sigma\right)(s, y)\right) p^{X^{\varepsilon}}(t, s, x, y) d y d s \\
& -\int_{\mathbf{R}^{d}} g(y)\left\{p^{\bar{X}}(t, T, x, y)+\sum_{i=1}^{N} \varepsilon^{i} E\left[\pi_{i, T}^{t, x} \mid \bar{X}_{T}^{t, x}=y\right] p^{\bar{X}}(t, T, x, y)\right\} d y \\
& -\int_{t}^{T} \int_{\mathbf{R}^{d}} f\left(s, y, u^{\varepsilon, k, N}(s, y),\left(\partial_{x} u^{\varepsilon, k, N} \sigma\right)(s, y)\right) \\
& \quad\left\{p^{\bar{X}}(t, s, x, y)+\sum_{i=1}^{N} \varepsilon^{i} E\left[\pi_{i, T}^{t, x} \mid \bar{X}_{s}^{t, x}=y\right] p^{\bar{X}}(t, s, x, y)\right\} d y d s \\
& \int_{\mathbf{R}^{d}} g(y) p^{X^{\varepsilon}}(t, T, x, y) d y-\int_{\mathbf{R}^{d}} g(y)\left\{p^{\bar{X}}(t, T, x, y)+\sum_{i=1}^{N} \varepsilon^{i} E\left[\pi_{i, T}^{t, x} \mid \bar{X}_{T}^{t, x}=y\right] p^{\bar{X}}(t, T, x, y)\right\} d y \\
& +\int_{t}^{T} \int_{\mathbf{R}^{d}} f\left(s, y, u^{\varepsilon, k}(s, y),\left(\partial_{x} u^{\varepsilon, k} \sigma\right)(s, y)\right) p^{X^{\varepsilon}}(t, s, x, y) d y d s \\
& -\int_{t}^{T} \int_{\mathbf{R}^{d}} f\left(s, y, u^{\varepsilon, k, N}(s, y),\left(\partial_{x} u^{\varepsilon, k, N} \sigma\right)(s, y)\right) p^{X^{\varepsilon}}(t, s, x, y) d y d s \\
& +\int_{t}^{T} \int_{\mathbf{R}^{d}} f\left(s, y, u^{\varepsilon, k, N}(s, y),\left(\partial_{x} u^{\varepsilon, k, N} \sigma\right)(s, y)\right) p^{X^{\varepsilon}}(t, s, x, y) d y d s \\
& -\int_{t}^{T} \int_{\mathbf{R}^{d}} f\left(s, y, u^{\varepsilon, k, N}(s, y),\left(\partial_{x} u^{\varepsilon, k, N} \sigma\right)(s, y)\right) \\
& \left\{p^{0}(t, s, x, y)+\sum_{i=1}^{N} \varepsilon^{i} E\left[\pi_{i, T}^{t, x} \mid \bar{X}_{s}^{t, x}=y\right] p^{\bar{X}}(t, s, x, y)\right\} d y d s .
\end{aligned}
$$


Remark that after the second equality, we add the terms

$$
\pm \int_{t}^{T} \int_{\mathbf{R}^{d}} f\left(s, y, u^{\varepsilon, k, N}(s, y),\left(\partial_{x} u^{\varepsilon, k, N} \sigma\right)(s, y)\right) p^{X^{\varepsilon}}(t, s, x, y) d y d s .
$$

Let $I_{1}, I_{2}$ and $I_{3}$ be

$$
\begin{aligned}
I_{1}(t, x):= & \int_{\mathbf{R}^{d}} g(y) p^{\varepsilon}(t, T, x, y) d y-\int_{\mathbf{R}^{d}} g(y)\left\{p^{\bar{X}}(t, T, x, y)+\sum_{i=1}^{N} \varepsilon^{i} E\left[\pi_{i, T}^{t, x} \mid \bar{X}_{T}^{t, x}=y\right] p^{\bar{X}}(t, T, x, y)\right\} d y, \\
I_{2}(t, x):= & \int_{t}^{T} \int_{\mathbf{R}^{d}} f\left(s, y, u^{\varepsilon, k}(s, y),\left(\partial_{x} u^{\varepsilon, k} \sigma\right)(s, y)\right) p^{X^{\varepsilon}}(t, s, x, y) d y d s \\
& -\int_{t}^{T} \int_{\mathbf{R}^{d}} f\left(s, y, u^{\varepsilon, k, N}(s, y),\left(\partial_{x} u^{\varepsilon, k, N} \sigma\right)(s, y)\right) p^{X^{\varepsilon}}(t, s, x, y) d y d s, \\
I_{3}(t, x):= & \int_{t}^{T} \int_{\mathbf{R}^{d}} f\left(s, y, u^{\varepsilon, k, N}(s, y),\left(\partial_{x} u^{\varepsilon, k, N} \sigma\right)(s, y)\right) p^{X^{\varepsilon}}(t, s, x, y) d y d s \\
& -\int_{t}^{T} \int_{\mathbf{R}^{d}} f\left(s, y, u^{\varepsilon, k, N}(s, y),\left(\partial_{x} u^{\varepsilon, k, N} \sigma\right)(s, y)\right) \\
& \left\{p^{\bar{X}}(t, s, x, y)+\sum_{i=1}^{N} \varepsilon^{i} E\left[\pi_{i, T}^{t, x} \mid \bar{X}_{s}^{t, x}=y\right] p^{\bar{X}}(t, s, x, y)\right\} d y d s .
\end{aligned}
$$

The difference $\left(\partial_{x} u^{\varepsilon, k+1} \sigma\right)-\left(\partial_{x} u^{\varepsilon, k+1, N} \sigma\right)$ is represented as

$$
\begin{aligned}
& \left(\partial_{x} u^{\varepsilon, k+1} \sigma\right)-\left(\partial_{x} u^{\varepsilon, k+1, N} \sigma\right) \\
= & \int_{\mathbf{R}^{d}} g(y) E\left[N_{T}^{\varepsilon, t, x} \mid X_{T}^{\varepsilon, t, x}=y\right] p^{X^{\varepsilon}}(t, T, x, y) d y \varepsilon \sigma(t, x) \\
& +\int_{t}^{T} \int_{\mathbf{R}^{d}} f\left(s, y, u^{\varepsilon, k}(s, y),\left(\partial_{x} u^{\varepsilon, k} \sigma\right)(s, y)\right) E\left[N_{s}^{\varepsilon, t, x} \mid X_{s}^{\varepsilon, t, x}=y\right] p^{X^{\varepsilon}}(t, s, x, y) d y d s \varepsilon \sigma(t, x) \\
& -\int_{\mathbf{R}^{d}} g(y) E\left[N_{0, T}^{t, x} \mid \bar{X}_{T}^{t, x}=y\right] p^{\bar{X}}(t, T, x, y) d y \varepsilon \sigma(t, x) \\
& -\sum_{i=1}^{N} \varepsilon^{i} \int_{\mathbf{R}^{d}} g(y) E\left[N_{i, T}^{t, x} \mid \bar{X}_{T}^{t, x}=y\right] p^{\bar{X}}(t, T, x, y) d y \varepsilon \sigma(t, x) \\
& -\int_{t}^{T} \int_{\mathbf{R}^{d}} f\left(s, y, u^{\varepsilon, k, N}(s, y),\left(\partial_{x} u^{\varepsilon, k, N} \sigma\right)(s, y)\right) E\left[N_{0, s}^{t, x} \mid \bar{X}_{s}^{t, x}=y\right] p^{\bar{X}}(t, s, x, y) d y d s \varepsilon \sigma(t, x) \\
& -\sum_{i=1}^{N} \varepsilon^{i} \int_{t}^{T} \int_{\mathbf{R}^{d}} f\left(s, y, u^{\varepsilon, k, N}(s, y),\left(\partial_{x} u^{\varepsilon, k, N} \sigma\right)(s, y)\right) E\left[N_{i, T}^{t, x} \mid \bar{X}_{T}^{t, x}=y\right] p^{\bar{X}}(t, T, x, y) d y d s \varepsilon \sigma(t, x) \\
& \int_{\mathbf{R}^{d}} g(y) E\left[N_{T}^{\varepsilon, t} \mid X_{T}^{\varepsilon, t, x}=y\right] p^{X^{\varepsilon}}(t, T, x, y) d y \varepsilon \sigma(t, x) \\
& -\int_{\mathbf{R}^{d}} g(y) E\left[N_{0, T}^{t, x} \mid \bar{X}_{T}^{t, x}=y\right] p^{\bar{X}}(t, T, x, y) d y \varepsilon \sigma(t, x) \\
& -\sum_{i=1}^{N} \varepsilon^{i} \int_{\mathbf{R}^{d}} g(y) E\left[N_{i, T}^{t, x} \mid \bar{X}_{T}^{t, x}=y\right] p^{\bar{X}}(t, T, x, y) d y \varepsilon \sigma(t, x) \\
& +\int_{t}^{T} \int_{\mathbf{R}^{d}} f\left(s, y, u^{\varepsilon, k}(s, y),\left(\partial_{x} u^{\varepsilon, k} \sigma\right)(s, y)\right) E\left[N_{s}^{\varepsilon, t, x} \mid X_{s}^{\varepsilon, t, x}=y\right] p^{X^{\varepsilon}}(t, s, x, y) d y d s \varepsilon \sigma(t, x) \\
& -\int_{t}^{T} \int_{\mathbf{R}^{d}} f\left(s, y, u^{\varepsilon, k, N}(s, y),\left(\partial_{x} u^{\varepsilon, k, N} \sigma\right)(s, y)\right) E\left[N_{s}^{\varepsilon, t, x} \mid X_{s}^{\varepsilon, t, x}=y\right] p^{X^{\varepsilon}}(t, s, x, y) d y d s \varepsilon \sigma(t, x) \\
& +\int_{t}^{T} \int_{\mathbf{R}^{d}} f\left(s, y, u^{\varepsilon, k, N}(s, y),\left(\partial_{x} u^{\varepsilon, k, N} \sigma\right)(s, y)\right) E\left[N_{s}^{\varepsilon, t} \mid X_{s}^{\varepsilon, t, x}=y\right] p^{X^{\varepsilon}}(t, s, x, y) d y d s \varepsilon \sigma(t, x) \\
& -\int_{t}^{T} \int_{\mathbf{R}^{d}} f\left(s, y, u^{\varepsilon, k, N}(s, y),\left(\partial_{x} u^{\varepsilon, k, N} \sigma\right)(s, y)\right) E\left[N_{0, s}^{t, x} \mid \bar{X}_{s}^{t, x}=y\right] p^{\bar{X}}(t, s, x, y) d y d s \varepsilon \sigma(t, x) \\
& -\sum_{i=1}^{N} \varepsilon^{i} \int_{t}^{T} \int_{\mathbf{R}^{d}} f\left(s, y, u^{\varepsilon, k, N}(s, y),\left(\partial_{x} u^{\varepsilon, k, N} \sigma\right)(s, y)\right) E\left[N_{i, s}^{t, x} \mid \bar{X}_{s}^{t, x}=y\right] p^{\bar{X}}(t, s, x, y) d y d s \varepsilon \sigma(t, x) . \\
& \\
&
\end{aligned}
$$


Let

$$
\begin{aligned}
& J_{1}(t, x) \\
:= & \int_{\mathbf{R}^{d}} g(y) E\left[N_{T}^{\varepsilon, t, x} \mid X_{T}^{\varepsilon, t, x}=y\right] p^{X^{\varepsilon}}(t, T, x, y) d y \varepsilon \sigma(t, x) \\
& -\int_{\mathbf{R}^{d}} g(y) E\left[N_{0, T}^{t, x} \mid \bar{X}_{T}^{t, x}=y\right] p^{\bar{X}}(t, T, x, y) d y \varepsilon \sigma(t, x) \\
& -\sum_{i=1}^{N} \varepsilon^{i} \int_{\mathbf{R}^{d}} g(y) E\left[N_{i, T}^{t, x} \mid \bar{X}_{T}^{t, x}=y\right] p^{\bar{X}}(t, T, x, y) d y \varepsilon \sigma(t, x), \\
& J_{2}(t, x) \\
:= & \int_{t}^{T} \int_{\mathbf{R}^{d}} f\left(s, y, u^{\varepsilon, k}(s, y),\left(\partial_{x} u^{\varepsilon, k} \sigma\right)(s, y)\right) E\left[N_{s}^{\varepsilon, t} \mid X_{s}^{\varepsilon, t, x}=y\right] p^{X^{\varepsilon}}(t, s, x, y) d y d s \varepsilon \sigma(t, x) \\
& -\int_{t}^{T} \int_{\mathbf{R}^{d}} f\left(s, y, u^{\varepsilon, k, N}(s, y),\left(\partial_{x} u^{\varepsilon, k, N} \sigma\right)(s, y)\right) E\left[N_{s}^{\varepsilon, t} \mid X_{s}^{\varepsilon, t, x}=y\right] p^{X^{\varepsilon}}(t, s, x, y) d y d s \varepsilon \sigma(t, x) \\
& J_{3}(t, x) \\
:= & \int_{t}^{T} \int_{\mathbf{R}^{d}} f\left(s, y, u^{\varepsilon, k, N}(s, y),\left(\partial_{x} u^{\varepsilon, k, N} \sigma\right)(s, y)\right) E\left[N_{s}^{\varepsilon, t, x} \mid X_{s}^{\varepsilon, t, x}=y\right] p^{X^{\varepsilon}}(t, s, x, y) d y d s \varepsilon \sigma(t, x) \\
& -\int_{t}^{T} \int_{\mathbf{R}^{d}} f\left(s, y, u^{\varepsilon, k, N}(s, y),\left(\partial_{x} u^{\varepsilon, k, N} \sigma\right)(s, y)\right) E\left[N_{0, s}^{t, x} \mid \bar{X}_{s}^{t, x}=y\right] p^{\bar{X}}(t, s, x, y) d y d s \varepsilon \sigma(t, x) \\
& -\sum_{i=1}^{N} \varepsilon^{i} \int_{t}^{T} \int_{\mathbf{R}^{d}} f\left(s, y, u^{\varepsilon, k, N}(s, y),\left(\partial_{x} u^{\varepsilon, k, N} \sigma\right)(s, y)\right) E\left[N_{i, s}^{t, x} \mid \bar{X}_{s}^{t, x}=y\right] p^{\bar{X}}(t, s, x, y) d y d s \varepsilon \sigma(t, x) .
\end{aligned}
$$

Then, we have

$$
\begin{gathered}
\left\|u^{\varepsilon, k+1}-u^{\varepsilon, k+1, N}\right\|_{H_{\beta, \mu}}^{2} \leq 3\left\|I_{1}\right\|_{H_{\beta, \mu}}^{2}+3\left\|I_{2}\right\|_{H_{\beta, \mu}}^{2}+3\left\|I_{3}\right\|_{H_{\beta, \mu}}^{2}, \\
\left\|\left(\partial_{x} u^{\varepsilon, k+1} \sigma\right)-\left(\partial_{x} u^{\varepsilon, k+1, N} \sigma\right)\right\|_{H_{\beta, \mu}}^{2} \leq 3\left\|J_{1}\right\|_{H_{\beta, \mu}}^{2}+3\left\|J_{2}\right\|_{H_{\beta, \mu}}^{2}+3\left\|J_{3}\right\|_{H_{\beta, \mu}}^{2} .
\end{gathered}
$$

By Proposition 5.1 and Proposition 5.2 and Lemma 5.2, we have the following estimates

$$
\begin{aligned}
\left|I_{1}(t, x)\right|= & \left|\int_{\mathbf{R}^{d}} g(y)\left\{p^{X^{\varepsilon}}(t, T, x, y)-p^{\bar{X}}(t, T, x, y)-\sum_{i=1}^{N} \varepsilon^{i} E\left[\pi_{i, T}^{t, x} \mid \bar{X}_{T}^{t, x}=y\right] p^{\bar{X}}(t, T, x, y)\right\} d y\right| \\
\leq & c(T, N, x) \varepsilon^{N+1}(T-t)^{(N+2) / 2}, \\
\left|J_{1}(t, x)\right|= & \mid \int_{\mathbf{R}^{d}} g(y)\left\{E\left[N_{T}^{\varepsilon, t, x} \mid X_{T}^{\varepsilon, t, x}=y\right] p^{X^{\varepsilon}}(t, T, x, y)\right. \\
& \left.-E\left[N_{0, T}^{t, x} \mid \bar{X}_{T}^{t, x}=y\right] p^{\bar{X}}(t, T, x, y)-\sum_{i=1}^{N} \varepsilon^{i} E\left[N_{i, T}^{t, x} \mid \bar{X}_{T}^{t, x}=y\right] p^{\bar{X}}(t, T, x, y)\right\} d y \varepsilon \sigma(t, x) \mid \\
\leq & r(T, N, x) \varepsilon^{N+1}(T-t)^{(N+1) / 2},
\end{aligned}
$$

and

$$
\begin{aligned}
\left|I_{3}(t, x)\right|= & \mid \int_{t}^{T} \int_{\mathbf{R}^{d}} f\left(s, y, u^{\varepsilon, k, N}(s, y),\left(\partial_{x} u^{\varepsilon, k, N} \sigma\right)(s, y)\right) \\
& \left\{p^{X^{\varepsilon}}(t, s, x, y)-p^{\bar{X}}(t, s, x, y)-\sum_{i=1}^{N} \varepsilon^{i} E\left[\pi_{i, s}^{t, x} \mid \bar{X}_{s}^{t, x}=y\right] p^{\bar{X}}(t, s, x, y)\right\} d y d s \mid \\
\leq & C(T, N, x) \varepsilon^{N+1} \int_{t}^{T}(s-t)^{(N+1) / 2} d s \\
= & C(T, N, x) \varepsilon^{N+1}(T-t)^{(N+3) / 2},
\end{aligned}
$$




$$
\begin{aligned}
\left|J_{3}(t, x)\right|= & \mid \int_{t}^{T} \int_{\mathbf{R}^{d}} f\left(s, y, u^{\varepsilon, k, N}(s, y),\left(\partial_{x} u^{\varepsilon, k, N} \sigma\right)(s, y)\right)\left\{E\left[N_{T}^{\varepsilon, t, x} \mid X_{T}^{\varepsilon, t, x}=y\right] p^{X^{\varepsilon}}(t, s, x, y)\right. \\
& \left.-E\left[N_{0, s}^{t, x} \mid \bar{X}_{s}^{t, x}=y\right] p^{\bar{X}}(t, s, x, y)-\sum_{i=1}^{N} \varepsilon^{i} E\left[N_{i, s}^{t, x} \mid \bar{X}_{s}^{t, x}=y\right] p^{\bar{X}}(t, s, x, y)\right\} d y d s \varepsilon \sigma(t, x) \mid \\
\leq & R(T, N, x) \varepsilon^{N+1} \int_{t}^{T}(s-t)^{N / 2} d s \\
= & R(T, N, x) \varepsilon^{N+1}(T-t)^{(N+2) / 2} .
\end{aligned}
$$

Here, $c(T, N, x), C(T, N, x), r(T, N, x)$ and $R(T, N, x)$ are some non-negative, non-decreasing and finite functions of at most polynomial growth in $x$ depending on $T$ and $N$.

Therefore, we obtain

$$
\begin{aligned}
& \left\|I_{1}\right\|_{H_{\beta, \mu}}^{2} \leq \varepsilon^{2(N+1)} K_{1}(T, N), \quad\left\|I_{3}\right\|_{H_{\beta, \mu}}^{2} \leq \varepsilon^{2(N+1)} K_{3}(T, N), \\
& \left\|J_{1}\right\|_{H_{\beta, \mu}}^{2} \leq \varepsilon^{2(N+1)} L_{1}(T, N), \quad\left\|J_{3}\right\|_{H_{\beta, \mu}}^{2} \leq \varepsilon^{2(N+1)} L_{3}(T, N),
\end{aligned}
$$

for some $K_{1}(T, N), K_{3}(T, N), L_{1}(T, N)$ and $L_{3}(T, N)$ depending on $T$ and $N$.

In order to estimate $\left\|I_{2}\right\|_{\beta, \mu}^{2}$ and $\left\|J_{2}\right\|_{\beta, \mu}^{2}$, we define

$$
\hat{u}^{\varepsilon, k+1}(t, x)=E\left[g\left(X_{T}^{\varepsilon, t, x}\right)\right]+E\left[\int_{t}^{T} f\left(s, X_{s}^{\varepsilon, t, x}, u^{\varepsilon, k, N}\left(s, X_{s}^{\varepsilon, t, x}\right),\left(\partial_{x} u^{\varepsilon, k, N} \sigma\right)\left(s, X_{s}^{\varepsilon, t, x}\right)\right) d s\right] .
$$

Since $f$ is Lipschitz countinuous with constant $C_{L}$, again using the norm equivalence result, (51) and the similar argument in the proof of Theorem 2.1 in El Karoui et al. [7] we obtain

$$
\begin{aligned}
& \left\|I_{2}\right\|_{H_{\beta, \mu}}^{2} \leq c_{1}^{-1}\left\|u^{\varepsilon, k+1}-\hat{u}^{\varepsilon, k+1}\right\|_{\beta, \mu, X^{\varepsilon}}^{2} \\
= & c_{1}^{-1} \int_{\mathbf{R}^{d}} \int_{0}^{T} e^{\beta s} E\left[\left|u^{\varepsilon, k+1}\left(s, X_{s}^{\varepsilon}\right)-\hat{u}^{\varepsilon, k+1}\left(s, X_{s}^{\varepsilon}\right)\right|^{2}\right] d s e^{-\mu|x|} d x \\
\leq & c_{1}^{-1} \frac{T}{\beta} \int_{\mathbf{R}^{d}} E\left[\int_{0}^{T} e^{\beta s} \mid f\left(s, X_{s}^{\varepsilon}, u^{\varepsilon, k}\left(s, X_{s}^{\varepsilon}\right), \partial_{x} u^{\varepsilon, k} \sigma\left(s, X_{s}^{\varepsilon}\right)\right)\right. \\
& \left.-\left.f\left(s, X_{s}^{\varepsilon}, u^{\varepsilon, k, N}\left(s, X_{s}^{\varepsilon}\right),\left(\partial_{x} u^{\varepsilon, k, N} \sigma\right)\left(s, X_{s}^{\varepsilon}\right)\right)\right|^{2} d s\right] e^{-\mu|x|} d x \\
\leq & \frac{2 c_{1}^{-1} C_{L}^{2} T}{\beta} \int_{\mathbf{R}^{d}} E\left[\int _ { 0 } ^ { T } e ^ { \beta s } \left\{\left|u^{\varepsilon, k}\left(s, X_{s}^{\varepsilon}\right)-u^{\varepsilon, k, N}\left(s, X_{s}^{\varepsilon}\right)\right|^{2}\right.\right. \\
\leq & \frac{2 C C_{L}^{2} T}{\beta}\left\{\left\|u^{\varepsilon, k}-u^{\varepsilon, k, N}\right\|_{H_{\beta, \mu}}^{2}+\left\|\left(\partial_{x} u^{\varepsilon, k} \sigma\right)-\left(\partial_{x} u^{\varepsilon, k, N} \sigma\right)\right\|_{H_{\beta, \mu}}^{2}\right\}, \\
& \left.\left.\left.\left\|J_{2}\right\|_{H_{\beta, \mu}}^{2} \leq c_{1}^{-1}\left\|\left(\partial_{x} u^{\varepsilon, k+1} \sigma\right)-\left(\partial_{x} \hat{u}^{\varepsilon, k+1} \sigma\right)\right\|_{\beta, \mu, X^{\varepsilon}}^{2}\right)-\left.\left(\partial_{x} u^{\varepsilon, k, N} \sigma\right)\left(s, X_{s}^{\varepsilon}\right)\right|^{2}\right\} d s\right] e^{-\mu|x|} d x \\
= & c_{1}^{-1} \int_{\mathbf{R}^{n}} \int_{0}^{T} e^{\beta s} E\left[\left|\left(\partial_{x} u^{\varepsilon, k+1} \sigma\right)\left(s, X_{s}^{\varepsilon}\right)-\left(\partial_{x} \hat{u}^{\varepsilon, k+1} \sigma\right)\left(s, X_{s}^{\varepsilon}\right)\right|^{2}\right] d s e^{-\mu|x|} d x \\
\leq & c_{1}^{-1} \frac{1}{\beta} \int_{\mathbf{R}^{d}} E\left[\int_{0}^{T} e^{\beta s} \mid f\left(s, X_{s}^{\varepsilon}, u^{\varepsilon, k}\left(s, X_{s}^{\varepsilon}\right),\left(\partial_{x} u^{\varepsilon, k} \sigma\right)\left(s, X_{s}^{\varepsilon}\right)\right)\right. \\
\leq & \frac{2 C C_{L}^{2}}{\beta}\left\{\left\|u^{\varepsilon, k}-u^{\varepsilon, k, N}\right\|_{H_{\beta, \mu}}^{2}+\left\|\left(\partial_{x} u^{\varepsilon, k} \sigma\right)-\left(\partial_{x} u^{\varepsilon, k, N} \sigma\right)\right\|_{H_{\beta, \mu}}^{2}\right\} . \\
\leq & \frac{2 c_{1}^{-1} C_{L}^{2}}{\beta} \int_{\mathbf{R}^{d}} E\left[\int _ { 0 } ^ { T } e ^ { \beta s } \left\{\left|u^{\varepsilon, k}\left(s, X_{s}^{\varepsilon}\right)-u^{\varepsilon, k, N}\left(s, X_{s}^{\varepsilon}\right)\right|^{2}\right.\right. \\
& \left.\left.+\left|\left(\partial_{x} u^{\varepsilon, k} \sigma\right)\left(s, X_{s}^{\varepsilon}\right)-\left(\partial_{x} u^{\varepsilon, k, N} \sigma\right)\left(s, X_{s}^{\varepsilon}\right)\right|^{2}\right\} d s\right] e^{-\mu|x|} d x \\
& \\
\leq &
\end{aligned}
$$


Then, we have the following estimates

$$
\begin{aligned}
& \left\|u^{\varepsilon, k+1}-u^{\varepsilon, k+1, N}\right\|_{H_{\beta, \mu}}^{2} \\
\leq & \varepsilon^{2(N+1)} K(T, N)+\frac{2 C C_{L}^{2} T}{\beta}\left\{\left\|u^{\varepsilon, k}-u^{\varepsilon, k, N}\right\|_{H_{\beta, \mu}}^{2}+\left\|\left(\partial_{x} u^{\varepsilon, k} \sigma\right)-\left(\partial_{x} u^{\varepsilon, k, N} \sigma\right)\right\|_{H_{\beta, \mu}}^{2}\right\}, \\
& \left\|\left(\partial_{x} u^{\varepsilon, k+1} \sigma\right)-\left(\partial_{x} u^{\varepsilon, k+1, N} \sigma\right)\right\|_{H_{\beta, \mu}}^{2} \\
\leq & \varepsilon^{2(N+1)} L(T, N)+\frac{2 C C_{L}^{2}}{\beta}\left\{\left\|u^{\varepsilon, k}-u^{\varepsilon, k, N}\right\|_{H_{\beta, \mu}}^{2}+\left\|\left(\partial_{x} u^{\varepsilon, k} \sigma\right)-\left(\partial_{x} u^{\varepsilon, k, N} \sigma\right)\right\|_{H_{\beta, \mu}}^{2}\right\},
\end{aligned}
$$

where $K(T, N)=2 \max \left\{K_{1}(T, N), K_{3}(T, N)\right\}$ and $L(T, N)=2 \max \left\{L_{1}(T, N), L_{3}(T, N)\right\}$. Therefore, by (58) and (59), we obtain

$$
\begin{aligned}
& \left\|u^{\varepsilon, k+1}-u^{\varepsilon, k+1, N}\right\|_{H_{\beta, \mu}}^{2}+\left\|\left(\partial_{x} u^{\varepsilon, k+1} \sigma\right)-\left(\partial_{x} u^{\varepsilon, k+1, N} \sigma\right)\right\|_{H_{\beta, \mu}}^{2} \\
\leq & \varepsilon^{2(N+1)} \gamma(T, N) \\
& +\frac{2 C C_{L}^{2}(T+1)}{\beta}\left\{\left\|u^{\varepsilon, k}-u^{\varepsilon, k, N}\right\|_{H_{\beta, \mu}}^{2}+\left\|\left(\partial_{x} u^{\varepsilon, k} \sigma\right)-\left(\partial_{x} u^{\varepsilon, k, N} \sigma\right)\right\|_{H_{\beta, \mu}}^{2}\right\},
\end{aligned}
$$

where $\gamma(T, N)=2 \max \{K(T, N), L(T, N)\}$.

Remark that the differences $u^{\varepsilon, 0}-u^{\varepsilon, 0, N}$ and $\partial_{x} u^{\varepsilon, 0} \sigma-\partial_{x} u^{\varepsilon, 0, N} \sigma$ are given as follows:

$$
\begin{aligned}
& u^{\varepsilon, 0}(t, x)-u^{\varepsilon, 0, N}(t, x) \\
= & \int_{\mathbf{R}^{d}} g(y) p^{X^{\varepsilon}}(t, T, x, y) d y \\
& \quad-\int_{\mathbf{R}} g(y)\left\{p^{\bar{X}}(t, T, x, y)+\sum_{i=1}^{N} \varepsilon^{i} E\left[\pi_{i, T}^{t, x} \mid \bar{X}_{T}^{t, x}=y\right] p^{\bar{X}}(t, T, x, y)\right\} d y \\
& +\int_{t}^{T} \int_{\mathbf{R}^{d}} f(s, y, 0,0) p^{X^{\varepsilon}}(t, s, x, y) d y d s \\
& -\int_{t}^{T} \int_{\mathbf{R}^{d}} f(s, y, 0,0)\left\{p^{\bar{X}}(t, s, x, y)+\sum_{i=1}^{N} \varepsilon^{i} E\left[\pi_{i, T}^{t, x} \mid \bar{X}_{s}^{t, x}=y\right] p^{\bar{X}}(t, s, x, y)\right\} d y d s
\end{aligned}
$$

and

$$
\begin{gathered}
\left(\partial_{x} u^{\varepsilon, 0} \sigma\right)(t, x)-\left(\partial_{x} u^{\varepsilon, 0, N} \sigma\right)(t, x) \\
=\int_{\mathbf{R}^{d}} g(y) E\left[N_{T}^{\varepsilon, t, x} \mid X_{T}^{\varepsilon, t, x}=y\right] p^{X^{\varepsilon}}(t, T, x, y) d y \varepsilon \sigma(t, x) \\
-\int_{\mathbf{R}^{d}} g(y) E\left[N_{0, T}^{t, x} \mid \bar{X}_{T}^{t, x}=y\right] p^{\bar{X}}(t, T, x, y) d y \varepsilon \sigma(t, x) \\
\quad-\sum_{i=1}^{N} \varepsilon^{i} \int_{\mathbf{R}^{d}} g(y) E\left[N_{i, T}^{t, x} \mid \bar{X}_{T}^{t, x}=y\right] p^{\bar{X}}(t, T, x, y) d y \varepsilon \sigma(t, x) \\
+\int_{t}^{T} \int_{\mathbf{R}^{d}} f(s, y, 0,0) E\left[N_{s}^{\varepsilon, t, x} \mid X_{s}^{\varepsilon, t, x}=y\right] p^{X^{\varepsilon}}(t, s, x, y) d y \varepsilon \sigma(t, x) \\
\quad-\int_{t}^{T} \int_{\mathbf{R}^{d}} f(s, y, 0,0) E\left[N_{0, s}^{t, x} \mid \bar{X}_{s}^{t, x}=y\right] p^{\bar{X}}(t, s, x, y) d y \varepsilon \sigma(t, x) \\
\quad-\sum_{i=1}^{N} \varepsilon^{i} \int_{t}^{T} \int_{\mathbf{R}^{d}} f(s, y, 0,0) E\left[N_{i, s}^{t, x} \mid \bar{X}_{s}^{t, x}=y\right] p^{\bar{X}}(t, s, x, y) d y d s \varepsilon \sigma(t, x) .
\end{gathered}
$$

Then, the term $\left\|u^{\varepsilon, 0}-u^{\varepsilon, 0, N}\right\|_{H_{\beta, \mu}}^{2}+\left\|\left(\partial_{x} u^{\varepsilon, 0} \sigma\right)-\left(\partial_{x} u^{\varepsilon, 0, N} \sigma\right)\right\|_{H_{\beta, \mu}}^{2}$ is estimated by the asymptotic error, that is,

$$
\left\|u^{\varepsilon, 0}-u^{\varepsilon, 0, N}\right\|_{H_{\beta, \mu}}^{2}+\left\|\left(\partial_{x} u^{\varepsilon, 0} \sigma\right)-\left(\partial_{x} u^{\varepsilon, 0, N} \sigma\right)\right\|_{H_{\beta, \mu}}^{2} \leq \varepsilon^{2(N+1)} K_{0}(T, N),
$$


for some $K_{0}(T, N)$.

Therefore, we obtain

$$
\begin{aligned}
& \left\|u^{\varepsilon, k+1}-u^{\varepsilon, k+1, N}\right\|_{H_{\beta, \mu}}^{2}+\left\|\left(\partial_{x} u^{\varepsilon, k+1} \sigma\right)-\left(\partial_{x} u^{\varepsilon, k+1, N} \sigma\right)\right\|_{H_{\beta, \mu}}^{2} \\
\leq & \varepsilon^{2(N+1)} C_{1}(T, N)+\frac{2 C C_{L}^{2}(T+1)}{\beta}\left\{\left\|u^{\varepsilon, k}-u^{\varepsilon, k, N}\right\|_{H_{\beta, \mu}}^{2}+\left\|\left(\partial_{x} u^{\varepsilon, k} \sigma\right)-\left(\partial_{x} u^{\varepsilon, k, N} \sigma\right)\right\|_{H_{\beta, \mu}}^{2}\right\} \\
\leq \quad & \varepsilon^{2(N+1)} C_{1}(T, N) \\
& +\frac{2 C C_{L}^{2}(T+1)}{\beta}\left\{\varepsilon^{2(N+1)} C_{1}(T, N)+\right. \\
& \left.\quad \frac{2 C C_{L}^{2}(T+1)}{\beta}\left\{\left\|u^{\varepsilon, k-1}-u^{\varepsilon, k-1, N}\right\|_{H_{\beta, \mu}}^{2}+\left\|\left(\partial_{x} u^{\varepsilon, k-1} \sigma\right)-\left(\partial_{x} u^{\varepsilon, k-1, N} \sigma\right)\right\|_{H_{\beta, \mu}}^{2}\right\}\right\} \\
\leq & \varepsilon^{2(N+1)} C_{1}(T, N)\left\{\left(\frac{2 C C_{L}^{2}(T+1)}{\beta}\right)^{k+1}+\cdots+\left(\frac{2 C C_{L}^{2}(T+1)}{\beta}\right)+1\right\} \\
= & \varepsilon^{2(N+1)} C_{1}(T, N) \cdot\left(\frac{1-\left(\frac{2 C C_{L}^{2}(T+1)}{\beta}\right)^{k+2}}{1-\left(\frac{2 C C_{L}^{2}(T+1)}{\beta}\right)}\right),
\end{aligned}
$$

where $C_{1}(T, N)=\max \left\{\gamma(T, N), K_{0}(T, N)\right\}$.

Finally, Choose $\beta$ such that $2 C C_{L}^{2}(T+1)<\beta$ and set $\delta=\frac{2 C C_{L}^{2}(T+1)}{\beta}<1$, by (52) and (61) we obtain the global error

$$
\left\|u^{\varepsilon}-u^{\varepsilon, k, N}\right\|_{H_{\beta, \mu}}^{2}+\left\|\left(\partial_{x} u^{\varepsilon} \sigma\right)-\left(\partial_{x} u^{\varepsilon, k, N} \sigma\right)\right\|_{H_{\beta, \mu}}^{2} \leq\left\{C_{0}(T) \cdot \delta^{k}+\varepsilon^{2(N+1)} C_{1}(T, N) \cdot\left(\frac{1-\delta^{k+1}}{1-\delta}\right)\right\} .
$$

\section{Application: pricing option with counterparty risk under local and stochastic volatility models}

This section applies our approximation algorithm to option pricing with counterparty risk in a FBSDE setting. Here, we omit a discussion on modeling and pricing issues under default risk, and concentrate on the concrete description of our approximation scheme with investigation of its validity by using a simple example. ${ }^{2}$ Particularly, we use local volatility and stochastic volatility models for the underlying (forward) price process $X$ under the risk-neutral measure. Let $Y$ be the solution to the following non-linear BSDE:

$$
Y_{t}=g\left(X_{T}\right)-(1-R) \beta \int_{t}^{T}\left(Y_{s}\right)^{+} d s-\int_{t}^{T} Z_{s} d W_{s} .
$$

Here, $Y$ represents the value process with a target payoff $g\left(X_{T}\right)$ taking the risky (substitution) closing out $C V A$ into account; $R \geq 0$ and $\beta>0$ denote a constant recovery rate and a constant default intensity, respectively. Also, the risk-free interest rate and the dividend rate of the underlying asset are assumed to be zero for simplicity. Next, let $\left(Y^{k}, Z^{k}\right)_{k \geq 0}$ be a sequence of the following linear BSDEs:

$$
\begin{aligned}
Y_{t}^{0} & =g\left(X_{T}\right)-\int_{t}^{T} Z_{s}^{0} d W_{s}^{1} . \\
Y_{t}^{1} & =g\left(X_{T}\right)-(1-R) \beta \int_{t}^{T}\left(Y_{s}^{0}\right)^{+} d s-\int_{t}^{T} Z_{s}^{1} d W_{s} . \\
Y_{t}^{k+1} & =g\left(X_{T}\right)-(1-R) \beta \int_{t}^{T}\left(Y_{s}^{k}\right)^{+} d s-\int_{t}^{T} Z_{s}^{k+1} d W_{s}, \quad k \geq 1,
\end{aligned}
$$

which is an approximation sequence of the value process $Y$.

Remark 6.1 Under the setting above, suppose we consider plain-vanilla options, that is $g\left(X_{T}\right)=\left(X_{T}-\right.$ $K)^{+}$or $\left(K-X_{T}\right)^{+}$. Then, given constant values of $R$ and $\beta$ as well as $Y^{k}>0$ for usual setup of

\footnotetext{
${ }^{2}$ See Fujii and Takahashi $(2010,2011)$ for the detail of modeling and pricing issues under default risk, for instance.
} 
parameters in practice, due to the martingale property of the (risk-free) option value $Y^{0}$ under the riskneutral measure, we are able to express $u^{k}(t, x):=Y_{t}^{k, t, x}$ for each $k=0,1,2, \cdots$ as follows:

$$
u^{k}(t, x)=u^{0}(t, x)\left[1+\sum_{i=1}^{k} \frac{q^{i}}{i !}\right],
$$

where $q=(-1)(1-R) \beta(T-t)$. Hence, for this simplest case we can easily obtain the benchmark values $u^{k}(t, x)$ through evaluation of $u^{0}(t, x)$ by numerical computation such as the Monte Carlo simulation, against which the validity of our approximation scheme is examined. However, note that it is much more tough task to get the benchmark values under the situation with stochastic intensity and recovery, while our scheme is applicable under the setting without substantial effort.

\subsection{Local volatility model}

First, we consider local volatility model (one dimensional process)

$$
d X_{t}^{\varepsilon}=\varepsilon \sigma\left(t, X_{t}^{\varepsilon}\right) d W_{t}, \quad X_{0}^{\varepsilon}=x_{0}
$$

where $\sigma(t, x)$ is the local volatility function. Define $u^{\varepsilon}(t, x):=Y_{t}^{\varepsilon, t, x}=E\left[g\left(X_{T}^{\varepsilon, t, x}\right)\right]-E\left[\int_{t}^{T}(1-R) \beta\left(Y_{s}^{\varepsilon, t, x}\right)^{+} d s\right]$. Then, $u^{0}(t, x):=Y_{t}^{\varepsilon, 0, t, x, 0}, u^{k+1}(t, x):=Y_{t}^{\varepsilon, k+1, t, x}, k \geq 0$, are approximated by

$$
\begin{aligned}
u^{0}(t, x) & \simeq u^{0, N}(t, x)=\int_{\mathbf{R}} g(y) p_{N}^{\bar{X}}(t, T, x, y) d y, \\
u^{k+1}(t, x) & \simeq u^{k+1, N}(t, x) \\
& =u^{0, N}(t, x)-(1-R) \beta \int_{t}^{T} \int_{\mathbf{R}}\left(u^{k, N}(\tau, y)\right)^{+} p_{N}^{\bar{X}}(t, \tau, x, y) d y d \tau, \quad k \geq 0,
\end{aligned}
$$

where $y \mapsto p_{N}^{\bar{X}}(t, s, x, y)$ is the $N$-th order asymptotic expansion of the density of $X_{s}^{\varepsilon, t, x}$. In our numerical example, we take $\varepsilon \sigma(t, x)=\varepsilon x_{0}^{1-\alpha} x^{\alpha}$ (CEV volatility). Here, $\varepsilon$ can be regarded as the instantaneous volatility of the log-normal (or the Black-Scholes) process. The terminal condition for the backward SDE is characterized as $g(x)=(x-K)^{+}$, the call option payoff function.

The parameters of the model are specified as follows:

$$
\begin{aligned}
& t=0.0, T=2.0, x_{0}=10,000, \quad \alpha=0.5, \varepsilon=0.1, \\
& \beta=0.06 \text { (intensity), } R=0.0 \text { (recovery rate). }
\end{aligned}
$$

Also, the expansion order $N$ is set to be $N=1$.

In this case, we can easily obtain $u^{0, N}(t, x)$ in $(66)$ as follows:

$$
u^{0, N}(t, x)=y N\left(\frac{y}{\sqrt{\Sigma(t, T)}}\right)+\left(\Sigma(t, T)-\frac{\zeta(t, T)}{\Sigma(t, T)} y\right) n[y: 0, \Sigma(t, T)]
$$

where $N(x)$ and $n[x: \mu, \Sigma]$ denote the standard normal distribution function, and the normal density function with the mean $\mu$ and the variance $\Sigma$, respectively. Also, $y, \Sigma(t, T)$ and $\zeta(t, T)$ are defined in the following:

$$
\begin{aligned}
y & =x-K, \\
\Sigma(t, T) & =\varepsilon^{2} \sigma^{2} x^{2 \alpha}(T-t), \\
\zeta(t, T) & =\alpha \varepsilon^{4} \sigma^{4} x^{4 \alpha-1} \frac{(T-t)^{2}}{2} .
\end{aligned}
$$

The result is given in Table 1-3: $\mathbf{A E} u^{k, N}\left(=u^{k, N}\left(0, x_{0}\right)\right),(k=0,1,2)$ are evaluated based on the corresponding equations in (67) and (68). Exact value $u\left(0, x_{0}\right)$ is approximated as in (64) by the equation (70) below with $k=5$, which gives the sufficiently convergent value for this case. Also, Benchmark $u^{k}=u^{k}\left(0, x_{0}\right), k=1,2$ are computed by the following equation (70) with $k=1,2$, respectively:

$$
u^{0}\left(0, x_{0}\right)\left[1+\sum_{i=1}^{k} \frac{q^{i}}{i !}\right]
$$


where $q=(-1)(1-R) \beta T$, and the value of $u^{0}\left(0, x_{0}\right)$ is obtained based on Monte Carlo simulation for the CEV process. In each simulation, the numbers of the trials and the time steps are 1,000,000 with the antithetic variable method and 750, respectively. Also, in Table 1-3 the relative errors denoted by AE Error $u$ and AE Error $u^{k}$ of our asymptotic expansion are computed by $\left(u^{k, N}\left(0, x_{0}\right)-u\left(0, x_{0}\right)\right) / u\left(0, x_{0}\right)$ and $\left(u^{k, N}\left(0, x_{0}\right)-u^{k}\left(0, x_{0}\right)\right) / u^{k}\left(0, x_{0}\right)$, respectively. It is observed that $u^{k, N}\left(=u^{k, N}\left(0, x_{0}\right)\right), k=1,2$, $N=1$ become closer towards $u\left(0, x_{0}\right)$.

Although this example use only the $\varepsilon^{1}$-order expansion of the density, we already know from the existing work (e.g. Takahashi et al. (2012)) that higher order expansions produce much more better approximation for the risk-free option price $u^{0}$, which is expected to provide more precise approximations for the solution to the BSDE as well.

Table 1: European call option price with CVA under CEV model (In-the-money case : $K=7500$, Exact value $\left.u\left(0, x_{0}\right)=2230.24\right)$

\begin{tabular}{|c|c|c|c|c|}
\hline Iteration $k$ & Benchmark $u^{k}$ & AE $u^{k, N}$ & AE Error $u$ & AE Error $u^{k}$ \\
\hline \hline 0th & 2514.59 & 2514.49 & $12.75 \%$ & $0.00 \%$ \\
1st & 2212.84 & 2212.81 & $-0.78 \%$ & $0.00 \%$ \\
2nd & 2230.41 & 2231.11 & $0.04 \%$ & $0.01 \%$ \\
\hline
\end{tabular}

Table 2: European call option price with CVA under CEV model (At-the-money case : $K=10000$, Exact value $\left.u\left(0, x_{0}\right)=499.45\right)$

\begin{tabular}{|c|c|c|c|c|}
\hline Iteration $k$ & Benchmark $u^{k}$ & AE $u^{k, N}$ & AE Error $u$ & AE Error $u^{k}$ \\
\hline \hline 0th & 563.13 & 564.19 & $12.96 \%$ & $0.19 \%$ \\
1st & 495.55 & 496.51 & $-0.59 \%$ & $0.19 \%$ \\
2nd & 499.61 & 500.61 & $0.23 \%$ & $0.20 \%$ \\
\hline
\end{tabular}

Table 3: European call option price with CVA under CEV model (Out-of-the-money case : $K=12500$, Exact value $\left.u\left(0, x_{0}\right)=26.01\right)$

\begin{tabular}{|c|c|c|c|c|}
\hline Iteration $k$ & Benchmark $u^{k}$ & AE $u^{k, N}$ & AE Error $u$ & AE Error $u^{k}$ \\
\hline \hline 0th & 29.33 & 29.28 & $12.55 \%$ & $-0.18 \%$ \\
1st & 25.81 & 25.76 & $-0.97 \%$ & $-0.20 \%$ \\
2nd & 26.02 & 25.97 & $-0.16 \%$ & $-0.20 \%$ \\
\hline
\end{tabular}

\subsection{Stochastic volatility model}

As an application, we consider a stochastic volatility model (SABR model):

$$
\begin{aligned}
d X_{t}^{\varepsilon} & =\varepsilon \sigma_{t}^{\varepsilon} C\left(t, X_{t}^{\varepsilon}\right) d W_{t}, \quad X_{0}^{\varepsilon}=x_{0}>0, \\
d \sigma_{t}^{\varepsilon} & =\varepsilon \sigma_{t}^{\varepsilon} d Z_{t}, \quad \sigma_{0}^{\varepsilon}=\sigma_{0}>0, \\
d W_{t} d Z_{t} & =\rho d t,
\end{aligned}
$$

where $C(t, x)$ is the local volatility function and $\rho \in[-1,1]$ is the correlation parameter. As in section 7.1, we put the terminal condition of the backward SDE as $g(x)=(x-K)^{+}$. Define $u(t, x, \sigma):=Y_{t}^{\varepsilon, t, x, \sigma}=$ $E\left[g\left(X_{T}^{\varepsilon, t, x, \sigma}\right)\right]-E\left[\int_{t}^{T}(1-R) \beta\left(Y_{s}^{\varepsilon, t, x, \sigma}\right)^{+} d s\right]$. Then, $u^{0}(t, x, \sigma):=Y_{t}^{\varepsilon, 0, t, x, \sigma}$ and $u^{k+1}(t, x, \sigma):=Y_{t}^{\varepsilon, k+1, t, x, \sigma}$, $k \geq 0$, are approximated by

$$
\begin{aligned}
u^{0}(t, x, \sigma) \simeq & u^{0, N}(t, x, \sigma)=E\left[g\left(\bar{X}_{T}^{t, x, \sigma}\right) \pi_{N, T}^{(X), t, x, \sigma}\right] . \\
u^{k+1}(t, x, \sigma) \simeq & u^{k+1, N}(t, x, \sigma) \\
= & E\left[g\left(\bar{X}_{T}^{t, x, a}\right) \pi_{N, T}^{(X), t, x, a}\right] \\
& -(1-R) \beta \int_{t}^{T} E\left[\left(u^{k, N}\left(\tau, \bar{X}_{\tau}^{t, x, \sigma}, \bar{\sigma}_{\tau}^{t, \sigma}\right)\right)^{+} \pi_{N, \tau}^{t, x, \sigma}\right] d \tau, \quad k \geq 0,
\end{aligned}
$$


where $\pi_{N, s}^{(X), t, x, \sigma}$ is the Malliavin weight of the $N$-th order expansion for the marginal $X_{s}^{\varepsilon, t, x, \sigma}$, and $\pi_{N, s}^{t, x, \sigma}$ is the Malliavin weight of the $N$-th order expansion for $\left(X_{s}^{\varepsilon, t, x}, \sigma_{s}^{\varepsilon, t, \sigma}\right)$. We take the local volatility function as $C(t, x)=c x_{0}^{1-\alpha} x^{\alpha}$.

The parameters of the model are specified as follows:

$$
\begin{aligned}
& t=0.0, T=1.0, x_{0}=100, \sigma_{0}=0.25, \alpha=0.5, \varepsilon=0.2, \varepsilon c=1, \\
& \rho=-0.5, \beta=0.05 \text { (intensity), } R=0.0 \text { (recovery rate) } .
\end{aligned}
$$

Also, the expansion order $N$ is set to be $N=1$.

Similarly as in the local volatility model, $u^{0, N}(t, x)$ is explicitly computed as follows:

$$
u^{0, N}(t, x, \sigma)=y N\left(\frac{y}{\sqrt{\Sigma(t, T)}}\right)+\left(\Sigma(t, T)-\frac{\zeta(t, T)}{\Sigma(t, T)} y\right) n[y: 0, \Sigma(t, T)],
$$

where $N(x)$ and $n[x: \mu, \Sigma]$ denote the standard normal distribution function, and the normal density function with the mean $\mu$ and the variance $\Sigma$, respectively. Also, $y, \Sigma(t, T)$ and $\zeta(t, T)$ are defined in the following:

$$
\begin{aligned}
y & =x-K \\
\Sigma(t, T) & =\varepsilon^{2} \sigma^{2} c^{2} x^{2 \alpha}(T-t) \\
\zeta(t, T) & =\left(\alpha \varepsilon^{4} \sigma^{4} c^{4} x^{4 \alpha-1}+\rho \varepsilon^{4} \sigma^{3} c^{3} x^{3 \alpha}\right) \frac{(T-t)^{2}}{2} .
\end{aligned}
$$

The result is given in Table $4-6$ similarly as in the local volatility case: $\mathbf{A E} u^{k, N}\left(=u^{k, N}\left(0, x_{0}, \sigma_{0}\right)\right)$ $(k=0,1,2, N=1)$ are evaluated based on the equations in (74) by applying the closed form approximation (76) and (75) by a numerical integration. Exact value $u\left(0, x_{0}, \sigma_{0}\right)$ is approximated by the method (70) for SABR model with iteration $k=5$. Also, Benchmark $u^{k}=u^{k}\left(0, x_{0}, \sigma_{0}\right), k=1,2$ are computed by $u^{0}\left(0, x_{0}, \sigma_{0}\right)\left[1+\sum_{i=1}^{k} \frac{q^{i}}{i !}\right]$ with $k=1,2$ where $q=(-1)(1-R) \beta T$, and the value of $u^{0}\left(0, x_{0}, \sigma_{0}\right)$ is obtained based on Monte Carlo simulation for the SABR process. In each simulation, the numbers of the trials and the time steps are 10,000,000 with the antithetic variable method and 1000, respectively. The relative errors in Table 4-6 are computed similarly as in local volatility case. We can observe that even low order expansions work well for numerical approximations of $u\left(0, x_{0}, \sigma_{0}\right)$. 
Table 4: European call option price with CVA under SABR model (In-the-money case : $K=70$, Exact value $\left.u\left(0, x_{0}, \sigma_{0}\right)=29.779\right)$

\begin{tabular}{|c|c|c|c|c|}
\hline Iteration $k$ & Benchmark $u^{k}$ & AE $u^{k, N}$ & AE Error $u$ & AE Error $u^{k}$ \\
\hline \hline 0th & 31.306 & 31.330 & $5.21 \%$ & $-0.08 \%$ \\
1st & 29.741 & 29.763 & $-0.05 \%$ & $-0.08 \%$ \\
2nd & 29.780 & 29.802 & $0.08 \%$ & $-0.08 \%$ \\
\hline
\end{tabular}

Table 5: European call option price with CVA under SABR model (At-the-money case : $K=100$, Exact value $\left.u\left(0, x_{0}, \sigma_{0}\right)=9.459\right)$

\begin{tabular}{|c|c|c|c|c|}
\hline Iteration $k$ & Benchmark $u^{k}$ & AE $u^{k, N}$ & AE Error $u$ & AE Error $u^{k}$ \\
\hline \hline 0th & 9.944 & 9.974 & $5.43 \%$ & $-0.29 \%$ \\
1st & 9.447 & 9.475 & $0.17 \%$ & $-0.29 \%$ \\
2nd & 9.460 & 9.488 & $0.30 \%$ & $-0.30 \%$ \\
\hline
\end{tabular}

Table 6: European call option price with CVA under SABR model (Out-of-the-money case : $K=130$, Exact value $\left.u\left(0, x_{0}, \sigma_{0}\right)=1.403\right)$

\begin{tabular}{|c|c|c|c|c|}
\hline Iteration $k$ & Benchmark $u^{k}$ & AE $u^{k, N}$ & AE Error $u$ & AE Error $u^{k}$ \\
\hline \hline 0th & 1.475 & 1.475 & $5.19 \%$ & $-0.08 \%$ \\
1st & 1.401 & 1.401 & $-0.08 \%$ & $-0.05 \%$ \\
2nd & 1.403 & 1.403 & $0.05 \%$ & $-0.05 \%$ \\
\hline
\end{tabular}

Remark 6.2 In the option valuation with CVA in FBSDE framework, we can easily obtain an approximation value for option price, only using the closed form approximation of clean price (76), as follows:

$$
u\left(0, x_{0}, \sigma_{0}\right) \simeq u^{0, N}\left(0, x_{0}, \sigma_{0}\right)\left[1+\sum_{i=1}^{k} \frac{q^{i}}{i !}\right], k \geq 1 .
$$

Actually, we have the following results using (78) with $k=2$ :

$$
\begin{aligned}
& (K=70) \quad \text { Benchmark: } 29.779, \text { Approximation using (78) : } 29.802 \text { (error } 0.079 \%), \\
& (K=100) \quad \text { Benchmark: } 9.459, \text { Approximation using (78):9.487 (error } 0.295 \%), \\
& (K=130) \quad \text { Benchmark: } 1.403, \text { Approximation using (78) }: 1.403 \text { (error } 0.059 \%) .
\end{aligned}
$$

Then, we can attain enough accuracy without using numerical methods such as Monte Carlo simulation or numerical integral in this case.

\section{Conclusion}

This paper has developed a new general approximation method for forward-backward stochastic differential equations (FBSDEs). In particular, we have proposed an analytical approximation based on an asymptotic expansion for forward SDEs combined with Picard-type iteration scheme for BSDEs. Based on the expansion with Malliavin calculus, we have justified our method with its error estimate for the approximation.

From a practical viewpoint, examination of our scheme under more complex examples is an important and interesting problem. Moreover, a challenging task is to develop mathematical validity of approximations with perturbation for fully coupled FBSDEs. Those topics as well as our approximation method under weaker mathematical condition will be discussed in our future researches. 


\section{A Proof of Lemma 5.1}

We prove the assertion by induction. First,

$$
\begin{aligned}
\frac{\partial}{\partial \varepsilon} X_{s}^{\varepsilon, t, x}= & \sum_{i=1}^{d} \int_{t}^{s} \partial_{x} X_{s}^{\varepsilon, t, x}\left(\partial_{x} X_{u}^{\varepsilon, t, x}\right)^{-1} \sigma_{i}\left(u, X_{u}^{\varepsilon, t, x}\right) d W_{u}^{i} \\
& +\varepsilon \sum_{i=1}^{d} \int_{t}^{s} \partial_{x} X_{s}^{\varepsilon, t, x}\left(\partial_{x} X_{u}^{\varepsilon, t, x}\right)^{-1} \partial_{x} \sigma_{i}\left(u, X_{u}^{\varepsilon, t, x}\right) \sigma_{i}\left(u, X_{u}^{\varepsilon}\right) d u .
\end{aligned}
$$

Since $\partial_{x} X_{s}^{\varepsilon, t, x},\left(\partial_{x} X_{s}^{\varepsilon, t, x}\right)^{-1} \in \mathcal{K}_{0}^{T}$, we have $\frac{\partial}{\partial \varepsilon} X_{s}^{\varepsilon, t, x} \in \mathcal{K}_{1}^{T}$.

For $k \geq 2, \frac{1}{k !} \frac{\partial^{k}}{\partial \varepsilon^{k}} X_{s}^{\varepsilon, t, x}=\left(\frac{1}{k !} \frac{\partial^{k}}{\partial \varepsilon^{k}} X_{s}^{\varepsilon, t, x, 1}, \cdots, \frac{1}{k !} \frac{\partial^{k}}{\partial \varepsilon^{k}} X_{s}^{\varepsilon, t, x, d}\right)$ is recursively determined by the following:

$$
\begin{aligned}
\frac{1}{k !} \frac{\partial^{k}}{\partial \varepsilon^{k}} X_{s}^{\varepsilon, t, x, j}= & \sum_{\mathbf{1}_{\beta}, \mathbf{d}_{\beta}}^{(k)} \int_{t}^{s}\left(\prod_{j=1}^{\beta} \frac{1}{l_{j} !} \frac{\partial^{l_{j}}}{\partial \varepsilon^{l_{j}}} X_{u}^{\varepsilon, t, x, d_{j}}\right) \partial_{d_{\beta}}^{\beta} b^{j}\left(u, X_{u}^{\varepsilon, t, x}\right) d u \\
& +\sum_{\mathbf{1}_{\beta}, \mathbf{d}_{\beta}}^{(k-1)} \int_{t}^{s}\left(\prod_{j=1}^{\beta} \frac{1}{l_{j} !} \frac{\partial^{l_{j}}}{\partial \varepsilon^{l_{j}}} X_{u}^{\varepsilon, t, x, d_{j}}\right) \sum_{i=1}^{d} \partial_{\mathbf{d}_{\beta}}^{\beta} \sigma_{i}^{j}\left(u, X_{u}^{\varepsilon, t, x}\right) d W_{u}^{i} \\
& +\varepsilon \sum_{\mathbf{1}_{\beta}, \mathbf{d}_{\beta}}^{(k)} \int_{t}^{u}\left(\prod_{j=1}^{k} \frac{1}{l_{j} !} \frac{\partial^{l_{j}}}{\partial \varepsilon^{l_{j}}} X_{u}^{\varepsilon, t, x, d_{j}}\right) \sum_{i=1}^{d} \partial_{\mathbf{d}_{k}}^{k} \sigma_{i}^{j}\left(u, X_{u}^{\varepsilon, t, x}\right) d W_{s}^{i}
\end{aligned}
$$

where $\partial_{d_{\beta}}^{\beta}=\frac{\partial^{\beta}}{\partial x_{d_{1}} \cdots \partial x_{d_{\beta}}}$,

$$
\sum_{\mathbf{1}_{\beta}, \mathbf{d}_{\beta}}^{(l)}:=\sum_{\beta=1}^{l} \sum_{\mathbf{1}_{\beta} \in L_{l, \beta}} \sum_{\mathbf{d}_{\beta} \in\{1, \cdots, d\}^{\beta}} \frac{1}{\beta !},
$$

and $L_{l, \beta}:=\left\{\mathbf{l}_{\beta}=\left(l_{1}, \cdots, l_{\beta}\right) ; \sum_{j=1}^{\beta} l_{j}=l ;\left(l, l_{j}, \beta \in \mathbf{N}\right)\right\}$. The above SDE is linear and the order of the Kusuoka function $\frac{1}{i !} \frac{\partial^{i}}{\partial \varepsilon^{i}} X_{s}^{\varepsilon, t, x}$ is determined inductively by the term

$$
\sum_{\mathbf{l}_{\beta}, \mathbf{d}_{\beta}}^{(i-1)} \frac{1}{\beta !} \int_{t}^{s} \partial X_{s}^{\varepsilon, t, x}\left(\partial X_{u}^{\varepsilon, t, x}\right)^{-1}\left(\prod_{j=1}^{\beta} \frac{1}{l_{j} !} \frac{\partial^{l_{j}}}{\partial \varepsilon^{l_{j}}} X_{u}^{\varepsilon, t, x, d_{j}}\right) \sum_{i=1}^{d} \partial_{\mathbf{d}_{\beta}}^{\beta} \sigma_{i}\left(u, X_{u}^{\varepsilon, t, x}\right) d W_{u}^{i} \in \mathcal{K}_{i}^{T} .
$$

Then, $\frac{1}{i !} \frac{\partial^{i}}{\partial \varepsilon^{i}} X_{s}^{\varepsilon, t, x} \in \mathcal{K}_{i}^{T}$.

\section{B Proof of Proposition 5.1}

Let $\left(\varphi_{n}\right)_{n \in \mathbf{N}} \subset C_{b}^{\infty}\left(\mathbf{R}^{d}\right)$ be a mollifier converging to $\varphi$. The following Taylor formula

$$
\varphi_{n}\left(F_{T}^{\varepsilon, t, x}\right)=\varphi_{n}\left(F_{T}^{0, t, x}\right)+\left.\sum_{i=1}^{N} \frac{\varepsilon^{i}}{i !} \frac{\partial^{i}}{\partial \varepsilon^{i}} \varphi_{n}\left(F_{T}^{\varepsilon, t, x}\right)\right|_{\varepsilon=0}+\left.\varepsilon^{N+1} \int_{0}^{1} \frac{(1-u)^{N}}{N !} \frac{\partial^{N+1}}{\partial \nu^{N+1}} \varphi_{n}\left(F_{T}^{\nu, t, x}\right)\right|_{\nu=\varepsilon u} d u,
$$

and the integration by parts on the Wiener space, we have

$$
\begin{aligned}
& E\left[\varphi_{n}\left(F_{T}^{\varepsilon, t, x}\right)\right]=E\left[\varphi_{n}\left(F_{T}^{0, t, x}\right)\right]+\sum_{i=1}^{N} \varepsilon^{i} \sum_{k}^{(i)} E\left[\partial_{\alpha^{(k)}} \varphi_{n}\left(F_{T}^{0, t, x}\right) \prod_{l=1}^{k} F_{\beta_{l}, T}^{0, t, x, \alpha_{l}}\right] \\
& +\varepsilon^{N+1} \int_{0}^{1}(1-u)^{N}(N+1) \sum_{k}^{(N+1)} E\left[\partial_{\alpha^{(k)}} \varphi_{n}\left(F_{T}^{\varepsilon u, t, x}\right) \prod_{l=1}^{k} F_{\beta_{l}, T}^{\varepsilon u, t, x, \alpha_{l}}\right] d u \\
= & E\left[\varphi_{n}\left(F_{T}^{0, t, x}\right)\right]+\sum_{i=1}^{N} \varepsilon^{i} E\left[\varphi_{n}\left(F_{T}^{0, t, x}\right) \pi_{i, T}^{t, x}\right] \\
& +\varepsilon^{N+1} \int_{0}^{1}(1-u)^{N}(N+1) \sum_{k}^{(N+1)} E\left[\partial_{\alpha^{(1)}} \varphi_{n}\left(F_{T}^{\varepsilon u, t, x}\right) H_{\alpha^{(k-1)}}\left(F_{T}^{\varepsilon u, t, x}, \prod_{l=1}^{k} F_{\beta_{l}, T}^{\varepsilon u, t, x, \alpha_{l}}\right)\right] d u,
\end{aligned}
$$


where, $\pi_{i, T}^{t, x}=\sum_{k}^{(i)} H_{\alpha^{(k)}}\left(F_{T}^{0, t, x}, \prod_{l=1}^{k} F_{\beta_{l}, T}^{0, t, x, \alpha_{l}}\right)=\sum_{k}^{(i)} H_{\alpha^{(k)}}\left(X_{1, T}^{0, t, x}, \prod_{l=1}^{k} X_{\beta_{l}+1, T}^{0, t, x, \alpha_{l}}\right)$.

Therefore, we have

$$
\begin{aligned}
& E\left[\varphi_{n}\left(X_{T}^{\varepsilon, t, x}\right)\right] \\
= & E\left[\varphi_{n}\left(\bar{X}_{T}^{t, x}\right)\right]+\sum_{i=1}^{N} \varepsilon^{i} E\left[\varphi_{n}\left(\bar{X}_{T}^{t, x}\right) \pi_{i, T}^{t, x}\right] \\
& +\varepsilon^{N+1} \int_{0}^{1}(1-u)^{N}(N+1) \sum_{k}^{(N+1)} E\left[\partial_{\alpha^{(1)}} \varphi_{n}\left(\tilde{X}_{T}^{\varepsilon u, t, x}\right) H_{\alpha^{(k-1)}}\left(F_{T}^{\varepsilon u, t, x}, \prod_{l=1}^{k} F_{\beta_{l}, T}^{\varepsilon u, t, x, \alpha_{l}}\right)\right] d u,
\end{aligned}
$$

where $\tilde{X}_{T}^{\varepsilon u, t, x}=X_{T}^{0, t, x}+\varepsilon F_{T}^{\varepsilon u, t, x}, u \in[0,1]$. By Proposition 4.1 with Lemma 4.1 and 5.1, we have $\sum_{k}^{(N+1)} H_{\alpha^{(k-1)}}\left(F_{T}^{\varepsilon u, t, x}, \prod_{l=1}^{k} F_{\beta_{l}, T}^{\varepsilon u, t, x, \alpha_{l}}\right) \in \mathcal{K}_{N+2}^{T}$.

Then, we obtain

$$
\left|E\left[\varphi_{n}\left(X_{T}^{\varepsilon, t, x}\right)\right]-E\left[\varphi_{n}\left(\bar{X}_{T}^{t, x}\right)\right]+\sum_{i=1}^{N} \varepsilon^{i} E\left[\varphi_{n}\left(\bar{X}_{T}^{t, x}\right) \pi_{i, T}^{t, x}\right]\right| \leq \varepsilon^{N+1}\left\|\nabla \varphi_{n}\right\|_{\infty}(T-t)^{(N+2) / 2} .
$$

Finally, by mollifier argument, we have the assertion.

\section{Proof of Proposition 5.2}

For a mollifier $\left(\varphi_{n}\right)_{n \in \mathbf{N}} \subset C_{b}^{\infty}\left(\mathbf{R}^{d}\right)$ converging to $\varphi$, we differentiate the expansion (89) of $E\left[\varphi_{n}\left(X_{T}^{\varepsilon, t, x}\right)\right]$ with respect to initial $x$ as follows: for $1 \leq \eta \leq d$,

$$
\begin{aligned}
& \frac{\partial}{\partial x_{\eta}} E\left[\varphi_{n}\left(X_{T}^{\varepsilon, t, x}\right)\right] \\
= & \frac{\partial}{\partial x_{\eta}} E\left[\varphi_{n}\left(\bar{X}_{T}^{t, x}\right)\right]+\sum_{i=1}^{N} \varepsilon^{i} \frac{\partial}{\partial x_{\eta}} E\left[\varphi_{n}\left(\bar{X}_{T}^{t, x}\right) \pi_{i, T}^{t, x}\right] \\
& +\varepsilon^{N+1} \int_{0}^{1}(1-u)^{N}(N+1) \sum_{k}^{(N+1)} \frac{\partial}{\partial x_{\eta}} E\left[\partial_{\alpha^{(1)}} \varphi_{n}\left(\tilde{X}_{T}^{\varepsilon u, t, x}\right) H_{\alpha^{(k-1)}}\left(F_{T}^{\varepsilon u, t, x}, \prod_{l=1}^{k} F_{\beta_{l}, T}^{\varepsilon u, t, x, \alpha_{l}}\right)\right] d u .
\end{aligned}
$$

We have

$$
\frac{\partial}{\partial x_{\eta}} E\left[\varphi_{n}\left(\bar{X}_{T}^{t, x}\right)\right]=\sum_{j=1}^{d} E\left[\partial_{j} \varphi_{n}\left(\bar{X}_{T}^{t, x}\right) \partial_{\eta} \bar{X}_{T}^{t, x, j}\right]=E\left[\varphi_{n}\left(\bar{X}_{T}^{t, x}\right) N_{0, T}^{t, x, \eta}\right],
$$

and, for $1 \leq i \leq N$,

$$
\begin{aligned}
\frac{\partial}{\partial x_{\eta}} E\left[\varphi_{n}\left(\bar{X}_{T}^{t, x}\right) \pi_{i, T}^{t, x}\right] & =\sum_{j=1}^{d}\left\{E\left[\partial_{j} \varphi_{n}\left(\bar{X}_{T}^{t, x}\right) \partial_{\eta} \bar{X}_{T}^{t, x, j} \pi_{i, T}^{t, x}\right]+E\left[\varphi_{n}\left(\bar{X}_{T}^{t, x}\right) \partial_{\eta} \pi_{i, T}^{t, x}\right]\right\} \\
& =E\left[\varphi\left(\bar{X}_{T}^{t, x}\right) N_{i, T}^{t, x, \eta}\right] .
\end{aligned}
$$

Moreover, $1 \leq \eta \leq d, u \in[0,1]$,

$$
\begin{aligned}
& \frac{\partial}{\partial x_{\eta}} E\left[\partial_{\alpha^{(1)}} \varphi_{n}\left(\tilde{X}_{T}^{\varepsilon u, t, x}\right) H_{\alpha^{(k-1)}}\left(F_{T}^{\varepsilon u, t, x}, \prod_{l=1}^{k} F_{\beta_{l}, T}^{\varepsilon u, t, x, \alpha_{l}}\right)\right] \\
&= \sum_{j=1}^{d} E\left[\partial_{j, \alpha^{(1)}} \varphi_{n}\left(\tilde{X}_{T}^{\varepsilon u, t, x}\right) \partial_{\eta} \tilde{X}_{T}^{\varepsilon u, t, x, j} H_{\alpha^{(k-1)}}\left(F_{T}^{\varepsilon u, t, x}, \prod_{l=1}^{k} F_{\beta_{l}, T}^{\varepsilon u, t, x, \alpha_{l}}\right)\right] \\
&+ E\left[\partial_{\alpha^{(1)}} \varphi_{n}\left(\tilde{X}_{T}^{\varepsilon u, t, x}\right) \partial_{\eta} H_{\alpha^{(k-1)}}\left(F_{T}^{\varepsilon u, t, x}, \prod_{l=1}^{k} F_{\beta_{l}, T}^{\varepsilon u, t, x, \alpha_{l}}\right)\right] \\
&= E\left[\partial _ { \alpha ^ { ( 1 ) } } \varphi _ { n } ( \tilde { X } _ { T } ^ { \varepsilon u , t , x } ) \left\{\sum_{j=1}^{d} H_{j}\left(\tilde{X}_{T}^{\varepsilon u, t, x}, \partial_{\eta} \tilde{X}_{T}^{\varepsilon u, t, x, j} H_{\alpha^{(k-1)}}\left(F_{T}^{\varepsilon u, t, x}, \prod_{l=1}^{k} F_{\beta_{l}, T}^{\varepsilon u, t, x, \alpha_{l}}\right)\right)\right.\right. \\
&\left.\left.+\partial_{\eta} H_{\alpha^{(k-1)}}\left(F_{T}^{\varepsilon u, t, x}, \prod_{l=1}^{k} F_{\beta_{l}, T}^{\varepsilon u, t, x, \alpha_{l}}\right)\right\}\right]
\end{aligned}
$$


where $\sum_{j=1}^{d} H_{j}\left(\tilde{X}_{T}^{\varepsilon u, t, x}, \partial_{\eta} \tilde{X}_{T}^{\varepsilon u, t, x, j} H_{\alpha^{(k-1)}}\left(F_{T}^{\varepsilon u, t, x}, \prod_{l=1}^{k} F_{\beta_{l}, T}^{\varepsilon u, t, x, \alpha_{l}}\right)\right)+\partial_{\eta} H_{\alpha^{(k-1)}}\left(F_{T}^{\varepsilon u, t, x}, \prod_{l=1}^{k} F_{\beta_{l}, T}^{\varepsilon u, t, x, \alpha_{l}}\right) \in$ $\mathcal{K}_{N+1}^{T}$. Therefore, we have the assertion.

\section{Proof of Lemma 5.2}

$u^{\varepsilon, 0, N}$ and $\partial_{x} u^{\varepsilon, 0, N} \sigma$ are represented as

$$
\begin{aligned}
u^{\varepsilon, 0, N}(t, x) & =E\left[g\left(\bar{X}_{T}^{t, x}\right) \vartheta_{T}\right]+E\left[\int_{t}^{T} f\left(s, \bar{X}_{s}^{t, x}, 0,0\right) \vartheta_{s} d s\right] \\
\partial_{x} u^{\varepsilon, 0, N} \sigma(t, x) & =\left\{E\left[g\left(\bar{X}_{T}^{t, x}\right) \gamma_{T}\right]+E\left[\int_{t}^{T} f\left(s, \bar{X}_{s}^{t, x}, 0,0\right) \gamma_{s} d s\right]\right\} \varepsilon \sigma(t, x),
\end{aligned}
$$

where $\vartheta_{s}=1+\sum_{i=1}^{N} \varepsilon^{i} \pi_{i, s}^{t, x}$ and $\gamma_{s}=\sum_{i=0}^{N} \varepsilon^{i} N_{i, s}^{t, x}$. Remark that $\vartheta_{s} \in \mathcal{K}_{\min \{0,1, \cdots, N\}}^{T}=\mathcal{K}_{0}^{T}$ and $\gamma_{s} \in$ $\mathcal{K}_{\min \{-1,0, \cdots, N-1\}}^{T}=\mathcal{K}_{-1}^{T}$. Since $g$ is Lipschitz continuous and of linear growth, we obtain

$$
\begin{aligned}
& \left|E\left[g\left(\bar{X}_{T}^{t, x}\right) \vartheta_{T}\right]\right| \leq\left\|g\left(\bar{X}_{T}^{t, x}\right)\right\|_{L^{p}}\left\|\vartheta_{T}\right\|_{L^{q}} \leq C(T, x), \\
& \left|E\left[g\left(\bar{X}_{T}^{t, x}\right) \gamma_{T}\right] \varepsilon \sigma(t, x)\right| \leq \varepsilon C_{L} C(T, x) .
\end{aligned}
$$

Also, as $f$ is of linear growth, we have

$$
\begin{aligned}
& \left|E\left[\int_{t}^{T} f\left(s, \bar{X}_{s}^{t, x}, 0,0\right) \vartheta_{s} d s\right]\right| \leq \int_{t}^{T} C(T, x) d s \\
& \left|E\left[\int_{t}^{T} f\left(s, \bar{X}_{s}^{t, x}, 0,0\right) \gamma_{s} d s\right] \varepsilon \sigma(t, x)\right| \leq \int_{t}^{T} C(T, x) \frac{1}{\sqrt{s-t}} d s,
\end{aligned}
$$

where $C(T, x)$ denotes a non-negative, non-decreasing and finite function of at most polynomial growth in $x$ depending on $T$. Then, we obtain estimates for $u^{\varepsilon, 0, N}$ and $\partial_{x} u^{\varepsilon, 0, N} \sigma$ :

$$
\begin{aligned}
\left|u^{\varepsilon, 0, N}(t, x)\right| & \leq C(T, x), \\
\left|\partial_{x} u^{\varepsilon, 0, N} \sigma(t, x)\right| & \leq C(T, x) .
\end{aligned}
$$

Note that for $k \geq 1$,

$$
\begin{aligned}
u^{\varepsilon, k, N}(t, x)= & E\left[g\left(\bar{X}_{T}^{t, x}\right) \vartheta_{T}\right] \\
& +E\left[\int_{t}^{T} f\left(s, \bar{X}_{s}^{t, x}, u^{\varepsilon, k-1, N}\left(s, \bar{X}_{s}^{t, x}\right), \partial_{x} u^{\varepsilon, k-1, N} \sigma\left(s, \bar{X}_{s}^{t, x}\right)\right) \vartheta_{s} d s\right], \\
\partial_{x} u^{\varepsilon, k, N} \sigma(t, x)=E\left[g\left(\bar{X}_{T}^{t, x}\right) \gamma_{T}\right] \varepsilon \sigma(t, x) & \\
& +E\left[\int_{t}^{T} f\left(s, \bar{X}_{s}^{t, x}, u^{\varepsilon, k-1, N}\left(s, \bar{X}_{s}^{t, x}\right), \partial_{x} u^{\varepsilon, k-1, N} \sigma\left(s, \bar{X}_{s}^{t, x}\right)\right) \gamma_{s} d s\right] \varepsilon \sigma(t, x),
\end{aligned}
$$

with (93), (94) and

$$
\begin{aligned}
& \left|E\left[\int_{t}^{T} f\left(s, \bar{X}_{s}^{0, t, x}, u^{\varepsilon, k-1, N}\left(s, \bar{X}_{s}^{t, x}\right), \partial_{x} u^{\varepsilon, k-1, N} \sigma\left(s, \bar{X}_{s}^{t, x}\right)\right) \vartheta_{s} d s\right]\right| \\
& \leq \int_{t}^{T} C(T, x) d s, \\
& \left|E\left[\int_{t}^{T} f\left(s, \bar{X}_{s}^{0, t, x}, u^{\varepsilon, k-1, N}\left(s, \bar{X}_{s}^{t, x}\right), \partial_{x} u^{\varepsilon, k-1, N} \sigma\left(s, \bar{X}_{s}^{t, x}\right)\right) \gamma_{s} d s\right] \varepsilon \sigma(t, x)\right| \\
& \leq \int_{t}^{T} C(T, x) \frac{1}{\sqrt{s-t}} d s .
\end{aligned}
$$

Then, recursively using (93), (94), (99) and (100) we obtain (49) and (50). 


\section{References}

[1] J.M. Bismut, Conjugate Convex Functions in Optimal Stochastic Control, J. Political Econ., 3, 637-654, (1973).

[2] R. Carmona (editor), Indifference Pricing, Princeton University Press, (2009)

[3] J. Cvitanic and I. Karatzas, Hedging Contingent Claims with Constrained Portfolios, The Annals of Applied Probability, 2, 652-681, (2003).

[4] J. Cvitanic, J. Ma and J. Zhang, Efficient Computation of Hedging Portfolios for Options with Discontinuous Payoffs, Mathematical Finance, (2003).

[5] D. Crisan and F. Delarue, Sharp Derivative Bounds for Solutions of Degenerate Semi-linear Partial Differential Equations, Journal of Functional Analysis, 263, 3024-3101, (2012).

[6] D. Crisan, K. Manolarakis and C. Nee, Cubature Methods and Applications, Paris-Princeton Lectures on Mathematical Finance 2013, Springer, (2013).

[7] N. El Karoui, Peng and Quenez, Backward Stochastic Differential Equations in Finance, Mathematical Finance, (1997).

[8] M. Fujii and A. Takahashi, Analytical Approximation for Non-Linear FBSDEs with Perturbation Scheme, International Journal of Theoretical and Applied Finance, Vol. 15, No. 5, (2012a).

[9] M. Fujii and A. Takahashi, Perturbative Expansion of FBSDE in an Incomplete Market with Stochastic Volatility, Quarterly Jornal of Finance Vol.2, No.3, (2012b).

[10] M. Fujii and A. Takahashi, Collateralized CDS and Default Dependence -Implications for the Central Clearing-, The Journal of Credit Risk, Vol.8-3, fall, (2012).

[11] M. Fujii and A. Takahashi, Derivative pricing under Asymmetric and Imperfect Collateralization and CVA, Quantitative Finance, Vol. 13, No.5, pp.749-768, (2013).

[12] M. Fujii and A. Takahashi, Perturbative Expansion Technique for Non-linear FBSDEs with Interacting Particle Method, Asia-Pacific Financial Markets, Vol 22, Issue 3, pp 283-304, (2015).

[13] M. Fujii, S. Sato and A. Takahashi, An FBSDE Approach to American Option Pricing with an Interacting Particle Method, Asia-Pacific Financial Markets, Vol 22-3, pp 239-260, (2015).

[14] E. Gobet and C. Labert, Solving BSDE with adaptive control variate, SIAM Journal on Numerical Analysis, Vol.48(1), pp.257-277, (2010).

[15] E. Gobet, J.-P. Lemor and X.A. Warin, Regression-based Monte Carlo method to solve backward stochastic differential equations, The Annals of Applied Probability, Vol.15(3), pp.2172-2002, (2005).

[16] N. Kunitomo and A. Takahashi, The Asymptotic Expansion Approach to the Valuation of Interest Rate Contingent Claims, Mathematical Finance, Vol.11, 117-151, (2001).

[17] N. Kunitomo and A. Takahashi, On Validity of the Asymptotic Expansion Approach in Contingent Claim Analysis, The Annals of Applied Probability, 13, no.3, 914-952, (2003).

[18] S. Kusuoka, Malliavin Calculus Revisited, J. Math. Sci. Univ. Tokyo, 261-277, (2003).

[19] S. Kusuoka and D. Stroock, Applications of the Malliavin Calculus Part I, Stochastic Analysis (Katata/Kyoto 1982) 271-306 (1984)

[20] C. Li, Managing Volatility Risk: Innovation of Financial Derivatives, Stochastic Models and Their Analytical Implementation, Ph.D thesis in Columbia University, (2010).

[21] C. Li, Closed-form Expansion, Conditional Expectation, and Option Valuation, Mathematics of Operations Research, Vol.39, Issue 2, pp.487-516, (2014).

[22] J. Ma and J. Yong, Forward-Backward Stochastic Differential Equations and their Applications, Springer, (2000).

[23] J. Ma and J. Zhang, Representation Theorems for Backward Stochastic Differential Equations, The Annals of Applied Probability, (2002).

[24] R. Matsuoka, A. Takahashi and Y. Uchida, A New Computational Scheme for Computing Greeks by the Asymptotic Expansion Approach, Asia-Pacific Financial Markets, Vol.11, pp.393-430, (2006).

[25] D. Nualart, The Malliavin Calculus and Related Topics, Springer, (2006).

[26] C. Nee, Lecture notes on Gradient bounds for Solutions of stochastic Differential Equations, Applications to numerical schemes, (2010). 
[27] C. Nee, Sharp Gradient Bounds for the Diffusion Semigroup, Ph.D thesis in Imperial College London, (2011).

[28] E. Pardoux and S. Peng, Adapted solution of a backward stochastic differential equation, Systems Control Lett. 14, no. 1, 55-61, (1990).

[29] K. Shiraya, A. Takahashi and T. Yamada Pricing Discrete Barrier Options Under Stochastic Volatility, Asia-Pacific Financial Markets, Vol 19 -3, pp 205-232, (2012)

[30] A. Takahashi, An Asymptotic Expansion Approach to Pricing Contingent Claims, Asia-Pacific Financial Markets, 6, 115-151, (1999).

[31] A. Takahashi, K. Takehara, An Asymptotic Expansion Approach to Currency Options with a Market Model of Interest Rates under Stochastic Volatility Processes of Spot Exchange Rates, Asia-Pacific Financial Markets, Vol.14-1,2, pp.69-121, (2007).

[32] A. Takahashi, K. Takehara and M. Toda, A General Computation Scheme for a High-Order Asymptotic Expansion Method, International Journal of Theoretical and Applied Finance, Vol.15-6, (2012).

[33] A. Takahashi. and T. Yamada, An Asymptotic Expansion with Push-Down of Malliavin Weights, SIAM Journal on Financial Mathematics, 3, 95-136, (2012).

[34] A. Takahashi. and T. Yamada, On Error Estimates for Asymptotic Expansions with Malliavin Weights -Application to Stochastic Volatility Models-, Mathematics of Operations Research, 40(3), 513-551, (2015).

[35] K. Yamamoto and A. Takahashi, A Remark on a Singular Perturbation Method for Option Pricing under a Stochastic Volatility Model, Asia-Pacific Financial Markets, Vol.16-4, pp.333-345, (2009). 